
\title{
$\begin{array}{ll}\text { Research Square } & \begin{array}{l}\text { Preprints are preliminary reports that have not undergone peer review. } \\ \text { They should not be considered conclusive, used to inform clinical practice, } \\ \text { or referenced by the media as validated information. }\end{array}\end{array}$
}

\section{The Role of Age Distribution, Time Lag Between Reporting and Death and Healthcare System Capacity in Case Fatality Estimates of COVID-19}

Patrizio Vanella ( $\sim$ patrizio.vanella@helmholtz-hzi.de)

Helmholtz Centre for Infection Research https://orcid.org/0000-0002-6736-6774

Christian Wiessner

University Medical Center Hamburg-Eppendorf

Anja Holz

University Medical Center Hamburg-Eppendorf

Gérard Krause

Helmholtz Centre for Infection Research

Annika Möhl

University Medical Center Hamburg-Eppendorf

Sarah Wiegel

University Medical Center Hamburg-Eppendorf

Berit Lange

Helmholtz Centre for Infection Research

Heiko Becher

University Medical Center Hamburg-Eppendorf

\section{Research}

Keywords: COVID-19 pandemic, Epidemiological Surveillance, Case Fatality Risk, Vulnerable Populations, Intensive Care, Healthcare, Public Health

Posted Date: July 1st, 2020

DOI: https://doi.org/10.21203/rs.3.rs-38592/v1

License: @) (1) This work is licensed under a Creative Commons Attribution 4.0 International License. Read Full License 


\section{Abstract}

Background: European countries report large differences in coronavirus disease (COVID-19) case fatality risk (CFR). CFR estimates depend on demographic characteristics of the cases, time lags between reporting of infections and deaths and infrastructural characteristics, such as healthcare and surveillance capacities.

Methods: We used publicly available data from official reports of the national health authorities of Germany, Italy, France, and Spain on COVID-19. These include age-specific numbers of cases and deaths for different dates, which we used to compute age-standardized CFR ratios using a standard European population for standardization. Moreover, we investigated the impact of different potential time lags on the estimation of the CFR using data published by the European Centre for Disease Prevention and Control (ECDC). Finally, we described the association between case fatality and the intensive care bed capacity.

Results: We found that age-standardized CFR estimates increased from the beginning of March to mid-May 2020 in all included European countries. In Germany, CFRs are lower than in other countries. However, the differences are much larger when comparing the crude risks rather than the age-adjusted risks. Thus, the different age distribution of the cases account for a major proportion of the reported differences. Case fatality estimates using time lags of 1-10 days converged in all countries over time, however, there is no optimal time lag to assess the CFR during the pandemic. Time lags that provided the most constant estimates and approach best the observed CFR after the pandemic ranged from 5-10 days in different countries and at different time points during the pandemic. For the association between intensive care bed capacity and fatality we found that days with a high need for intensive care beds were positively correlated with daily hospitalization fatality in France, Italy, and Spain, but not in Germany.

Conclusions: Our results highlight that cross-country comparisons of crude CFR estimates can be misleading and should be avoided. However, to adjust for potential sources of bias more disaggregated data and information on surveillance and health care capacities are needed. Filling these gaps and harmonizing data across European countries will facilitate further analysis.

\section{Background}

Different case fatalities of COVID-19 have been reported in affected countries. Until May 14th, 2020, over 4.3 million confirmed cases and close to 300,000 associated deaths have been identified globally (1). The crude case fatality risk (CFR) estimate, namely the cumulative number of deaths divided by the cumulative number of cases, is known to be biased (2). The main sources of bias are given in Table 1. A distinction must be made between factors that influence the actual lethality (as denoted by ${ }^{*}$ ), such as healthcare capacity and those that bias the estimates of the CFR, such as an underassessment of cases.

Table 1

Sources of bias of case fatality risk estimates

\begin{tabular}{|c|c|c|c|}
\hline Factor & Description & Impact on CFR estimate & Literature \\
\hline $\begin{array}{l}\text { Population } \\
\text { structure }^{*}\end{array}$ & $\begin{array}{l}\text { Age, comorbidities and underlying } \\
\text { risk factors }\end{array}$ & Higher CFR given an older population with a higher load of comorbidities & $(3-11)$ \\
\hline \multirow[t]{3}{*}{$\begin{array}{l}\text { Surveillance } \\
\text { and testing }\end{array}$} & $\begin{array}{l}\text { Surveillance system and different } \\
\text { testing capacities }\end{array}$ & $\begin{array}{l}\text { Overestimation of CFR given a poor surveillance system and poor testing capacity, } \\
\text { as fewer currently infected persons in relation to deaths are counted }\end{array}$ & $\begin{array}{l}(2,12- \\
19)\end{array}$ \\
\hline & \multirow[t]{2}{*}{$\begin{array}{l}\text { Methods and capacities to record } \\
\text { deaths }\end{array}$} & $\begin{array}{l}\text { Underestimation of CFR given low capacities and poor quality methods to record } \\
\text { deaths due to the disease resulting in a smaller numerator of deaths / current } \\
\text { reported infected; }\end{array}$ & $(20)$ \\
\hline & & $\begin{array}{l}\text { Overestimation of CFR, if all deaths are counted regardless of whether the patient } \\
\text { died of the target disease or another cause given the same number of } \\
\text { infected/diseased }\end{array}$ & $(20)$ \\
\hline Time lag & $\begin{array}{l}\text { Deaths occur time-delayed after } \\
\text { infection }\end{array}$ & $\begin{array}{l}\text { Underestimation of CFR given a time lag of several days between case registration } \\
\text { and death resulting in a smaller nominator of current deaths / current infected }\end{array}$ & $(11,21)$ \\
\hline $\begin{array}{l}\text { Healthcare } \\
\text { system }^{*}\end{array}$ & $\begin{array}{l}\text { Healthcare system capacity } \\
\text { measured as number of intensive } \\
\text { care beds per } 100.000 \text { inhabitants }\end{array}$ & $\begin{array}{l}\text { Higher CFR given a low healthcare capacity and an additional excessive demand for } \\
\text { intensive care beds during the pandemic resulting in more deaths and therefore a } \\
\text { higher numerator of deaths / current infected }\end{array}$ & $\begin{array}{l}(4,17 \\
22-26)\end{array}$ \\
\hline
\end{tabular}

Different demographic characteristics of infected cases regarding age, comorbidities, or underlying risk factors, as well as different underlying population structures of the respective countries, might explain different CFRs. There is increasing evidence that old-age and comorbidities - such as hypertension, diabetes, cardiovascular disease, or chronic lung disease - are major risk factors for severe COVID-19 infection outcomes (6, 27, 28). The higher susceptibility to disease as well as the higher prevalence of comorbidities in the elderly has an impact on the morbidity as well as mortality of this subpopulation resulting in higher CFRs in countries with an older population compared to countries with a younger age structure, which is apparent in the COVID-19 pandemic (3-10).

Different surveillance systems and different testing capacities across countries lead to huge variations in the number of tests performed $(12,13,16,17,29)$. Underassessment of reported infections therefore differs among countries $(14,15)$. Furthermore, surveillance and testing capacities influence the probability of detecting infections early and thus to enact countermeasures. Capacities and methods to record deaths as having been caused by COVID-19 also differ between countries (20). While in some countries, post-mortem screening of all deaths has been installed, other countries are only performing this when there is clinical suspicion (30). 
There exists a time lag between reporting of an infection and eventual death of said individual. The distribution of the time lag may differ between countries. This delay is not reflected in crude CFR estimates (21). A more robust estimate would be given by dividing cumulative deaths by cumulative recoveries. This, however, is not a reliable estimate as well due to a low number of recoveries during the early stages of the pandemic, when a high relative increase of infection numbers and an incomplete reporting of recoveries are witnessed (31). Therefore, some authors propose to investigate the cumulative deaths in relation to lags of various days of the cumulative infection numbers $(21,31)$. However, as a result of the high transmission rates of the virus in the early stages of the epidemic, the estimates depend strongly on the appropriate lag, and both under- and overestimation of the true CFR can occur (32). Gianicolo et al. (11) calculate age-standardized CFRs with a time lag of 7 days ranging from $0.8 \%$ in South Korea to $2.4 \%$ in Italy. In contrast, they report crude CFRs of $2.2 \%$ for South Korea and $12.6 \%$ for Italy.

Furthermore, CFRs are influenced by the healthcare system capacity of the affected countries. Previous studies have shown, that healthcare capacities differ substantially among countries, and even among regions within countries $(4,17,22-26)$. A healthcare system overwhelmed by the pandemic may result in higher CFRs.

All factors mentioned above may explain differences in CFRs in affected countries to a certain degree at different time points during the COVID-19 pandemic. It is unclear, how much of the difference in during-epidemic CFR estimates is explained by each of these factors. The aims of this paper are as follows: (i) to quantify the difference in during-epidemic case fatality due to COVID-19 between the countries Germany, Italy, France, and Spain attributed to differences in the age structures among the reported cases, (ii) to investigate the time lag between case reporting and death and its effect on CFR estimation, and (iii) to discuss the association between CFR and the healthcare system capacity. The selected countries are interesting for our study, as they are the four most populous countries in the European Union (EU), covering more than half of the total population of the EU (33). Moreover, these countries show different levels in COVID-19 CFRs and have born a large part of the COVID-19 disease burden over the study period.

\section{Data And Methods}

To investigate the age effect, we obtained the cumulative numbers of cases and deaths of Germany $(34)$, Italy $(35-38)$, France (39, 40), and Spain $(41)$ by age group for different dates as provided from the websites of the national health authorities. For comparison of crude and age-adjusted CFRs, we used the European Standard Population (42) for standardization. The effect of different time lags on CFR estimates was analyzed using data provided daily by the European Centre for Disease Prevention and Control (ECDC) (43). For our investigation of the impact of the healthcare system capacity, we used estimates for the available critical care beds from the OECD (44) and of needed critical care beds for COVID-19 patients on a daily basis from the Institute for Health Metrics and Evaluation (IHME) (45-48).

We use the following notation: $n_{i j k}$ and $d_{i j k}$ denote the total cumulative number of cases and deaths, respectively, for age group i, country j, and up to day k. $\delta_{\cdot j k}$ denotes the number of deaths at day k, i.e. $d_{. j k}=\sum_{K=1}^{k} \delta_{j K}$.

Figure 1 illustrates the development of the crude CFR estimates of the four selected countries in percent between March 4th (i.e., calendar week 10) and May 14th (i.e., calendar week 20), 2020 as obtained by $C F R_{j k}=d_{. j k} / n_{. j k}$. These crude estimates do not take into account the factors listed in Table 1.

Figure 1. Crude Case Fatality Risk Estimates due to COVID-19 between March 4th and May 14th, 2020 Sources: (43); Own computation and design

All curves increased over the study period. We observed significant differences between the curves. Our study aims to explain these differences to some extent.

The first aim was to identify the role of the cases' age structure in the overall CFRs and to derive age-specific and age-standardized CFR estimates of Germany, Italy, France, and Spain. Based on the age-specific cases $\mathrm{n}_{\mathrm{ijk}}$ of the four European countries, we calculated the age-specific CFR, $C F R_{i j k}=d_{i j k} / n_{i j k}$, the total crude CFR estimate, $C F R_{j k}=d_{. j k} / n_{. j k}$ and the age-standardized CFR estimate as $C F R_{j k}=\sum_{i} w_{i} \times d_{i j k} / n_{i j k}$ using the European Standard Population as a reference. To illustrate the relative effect of the different age distributions of cases in the countries considered, we calculated the ratio of the relative differences between the crude and age-adjusted CFR estimates, using Germany as the reference country, as it is the country with the lowest crude CFRs.

For the second aim, namely, to investigate the effect of different lags on the CFR estimate, the unknown distribution of the time lag $\Delta$ between reporting of a case and death was considered. Verity et al. (49) estimated the average time from infection to death at about 14 days. Thus, the average time from reporting a case to death is several days shorter. The effect of $\Delta$ on the CFR estimate was evaluated by calculating $C F R_{k, \Delta}=d_{. j k} / n{ }_{. j k-\Delta}$ for the pandemic for $k=0$ over the study period with $\Delta=0, \ldots, 10$. We graphically determined the values $\tilde{\Delta}$, which appear to converge to the final CFR of the epidemic over time, in particular during the peak of the epidemic. We then present the figures for Germany, Italy, Spain and France.

The third aim was to investigate the association between the daily hospitalization fatality and the healthcare capacity of a specific country. As a suitable measure for the daily hospitalization fatality, we suggest the number of COVID-19 related fatalities of a particular day divided by the number of hospital admissions due to COVID-19 of the last 14 days:

$$
\text { Dailyhospitalizationfatality }_{j k}=\frac{\delta_{j k}}{\sum_{K=k-14}^{k} h_{j K}}
$$

A period of the preceding two weeks was used to (a) remove the weekly periodicity and (b) because of a reported median hospitalization length of stay of 8 days $(50,51)$. As a measure of the utilization of healthcare capacities, the number of intensive care beds needed for COVID-19 patients was chosen. The 
analysis only included days where at least $5 \%$ of the total number of intensive care beds of a country were needed for COVID-19 patients because with lower numbers no overload of the intensive care bed capacity could be expected (44).

We then related the daily hospitalization fatality to the number of intensive care beds needed (45-48). The total number of intensive care beds available is included in Fig. 4 as a threshold, above which demand exceeded capacity.

\section{Results}

Aim 1. During-epidemic crude and age-standardized CFR estimates, alongside CFR ratios of Germany, Italy, France and Spain

Table 2 provides weekly estimates of crude and age-standardized CFRs, estimated as explained in the section Data and Methods. Moreover, the standardized CFRs are normalized to Germany's age-standardized CFR as the baseline, to investigate how much higher the CFRs were in Italy, France and Spain compared to Germany after accounting for the age structure of the cases. 
Table 2

Crude and standardized CFRs in \% for Germany, Italy, France and Spain by calendar week 2020

\begin{tabular}{|c|c|c|c|c|c|}
\hline \multirow[t]{2}{*}{ Week } & \multirow[t]{2}{*}{ Variable } & \multicolumn{4}{|l|}{ Country } \\
\hline & & Germany & Italy & France & Spain \\
\hline \multirow[t]{4}{*}{20} & CFR (crude) & 4.46 & 13.37 & 19.25 & 10.76 \\
\hline & ratio & 1.0 (reference) & 3.00 & 4.32 & 2.41 \\
\hline & CFR (age-standardized) & 3.19 & 5.55 & 6.82 & 3.87 \\
\hline & ratio & 1.0 (reference) & 1.74 & 2.14 & 1.21 \\
\hline \multirow[t]{4}{*}{19} & CFR (crude) & 4.24 & 13.06 & 19.22 & 11.77 \\
\hline & ratio $^{2}$ & 1.0 (reference) & 3.08 & 4.53 & 2.78 \\
\hline & CFR (age-standardized) & 3.04 & 5.41 & 6.58 & 3.73 \\
\hline & ratio $^{3}$ & 1.0 (reference) & 1.78 & 2.16 & 1.23 \\
\hline \multirow[t]{4}{*}{18} & CFR (crude) & 3.88 & 12.64 & 18.67 & 11.50 \\
\hline & ratio $^{2}$ & 1.0 (reference) & 3.26 & 4.81 & 2.96 \\
\hline & CFR (age-standardized) & 2.80 & 5.24 & 6.35 & 3.62 \\
\hline & ratio $^{3}$ & 1.0 (reference) & 1.87 & 2.27 & 1.29 \\
\hline \multirow[t]{4}{*}{17} & CFR (crude) & 3.35 & 13.09 & 17.73 & 10.40 \\
\hline & ratio $^{2}$ & 1.0 (reference) & 3.91 & 5.29 & 3.10 \\
\hline & CFR (age-standardized) & 2.59 & 5.44 & 6.03 & 4.03 \\
\hline & ratio $^{3}$ & 1.0 (reference) & 2.10 & 2.33 & 1.56 \\
\hline \multirow[t]{4}{*}{16} & CFR (crude) & 2.55 & 12.57 & 16.08 & 10.57 \\
\hline & ratio $^{2}$ & 1.0 (reference) & 4.93 & 6.31 & 4.15 \\
\hline & CFR (age-standardized) & 1.8 & 5.25 & 5.48 & 3.86 \\
\hline & ratio $^{3}$ & 1.0 (reference) & 2.92 & 3.05 & 2.14 \\
\hline \multirow[t]{4}{*}{15} & CFR (crude) & 1.8 & 12.24 & 10.89 & 9.92 \\
\hline & ratio $^{2}$ & 1.0 (reference) & 6.80 & 6.05 & 5.51 \\
\hline & CFR (age-standardized) & 1.41 & 5.16 & 4.84 & 2.65 \\
\hline & ratio ${ }^{3}$ & 1.0 (reference) & 3.66 & 3.43 & 1.88 \\
\hline \multirow[t]{4}{*}{14} & CFR (crude) & 1.09 & 11.80 & 9.83 & 9.19 \\
\hline & ratio $^{2}$ & 1.0 (reference) & 10.83 & 9.02 & 8.43 \\
\hline & CFR (age-standardized) & 1.05 & 4.90 & 3.70 & 3.01 \\
\hline & ratio $^{3}$ & 1.0 (reference) & 4.66 & 3.52 & 2.87 \\
\hline \multirow[t]{4}{*}{13} & CFR (crude) & 0.47 & 9.22 & 4.93 & 7.71 \\
\hline & ratio $^{2}$ & 1.0 (reference) & 19.62 & 10.49 & 16.40 \\
\hline & CFR (age-standardized) & 0.56 & 3.78 & 2.02 & 1.73 \\
\hline & ratio ${ }^{3}$ & 1.0 (reference) & 6.75 & 3.60 & 3.09 \\
\hline 12 & CFR (crude) & 0.15 & 8.53 & 2.52 & 0.05 \\
\hline
\end{tabular}

Sources: (34-43); Own computation and design

Trends in all countries examined are similar, with higher age-specific risks in older age groups. At the end of our study period, age-specific case fatalities for persons above the age of 79 were over one fifth in all study countries, whereas age-specific fatality for those under 60 years of age was consistently below $5 \%$ in all countries (see Tables 3-6 in the Appendix).

Figure 2. Crude and age-standardized CFR estimates Sources: (34-42); own computation and design

Until mid-May, the proportion of cases exceeding 60 years of age (among those with known age) was above $50 \%$ in Italy, Spain and France, compared to approximately one third in Germany, yet the trend in Germany was positive. For Germany, the age-standardized CFR estimates show a smaller increase than the crude CFRs over time, caused by the changing age distribution of the cases towards a higher proportion of older cases. 


\begin{tabular}{|c|c|c|c|c|c|}
\hline & ratio $^{2}$ & 1.0 (reference) & 56.87 & 16.80 & 0.33 \\
\hline & CFR (age-standardized) & 0.23 & 3.42 & 1.35 & 0.00 \\
\hline & ratio ${ }^{3}$ & 1.0 (reference) & 14.87 & 5.89 & - \\
\hline \multirow[t]{4}{*}{11} & CFR (crude) & 0.19 & 5.36 & 2.21 & 0.03 \\
\hline & ratio $^{2}$ & 1.0 (reference) & 28.21 & 11.63 & 0.16 \\
\hline & CFR (age-standardized) & 0.68 & 1.93 & 1.60 & 0.00 \\
\hline & ratio ${ }^{3}$ & 1.0 (reference) & 2.84 & 2.35 & - \\
\hline \multicolumn{6}{|c|}{ Sources: (34-43); Own computation and design } \\
\hline \multicolumn{6}{|c|}{$\begin{array}{l}\text { Trends in all countries examined are similar, with higher age-specific risks in older age groups. At the end of our study period, age-specific case fatalities } \\
\text { for persons above the age of } 79 \text { were over one fifth in all study countries, whereas age-specific fatality for those under } 60 \text { years of age was consistently } \\
\text { below } 5 \% \text { in all countries (see Tables } 3-6 \text { in the Appendix). }\end{array}$} \\
\hline \multicolumn{6}{|c|}{ Figure 2. Crude and age-standardized CFR estimates Sources: (34-42); own computation and design } \\
\hline \multicolumn{6}{|c|}{$\begin{array}{l}\text { Until mid-May, the proportion of cases exceeding } 60 \text { years of age (among those with known age) was above } 50 \% \text { in Italy, Spain and France, compared to } \\
\text { approximately one third in Germany, yet the trend in Germany was positive. For Germany, the age-standardized CFR estimates show a smaller increase } \\
\text { than the crude CFRs over time, caused by the changing age distribution of the cases towards a higher proportion of older cases. }\end{array}$} \\
\hline
\end{tabular}


Table 3

Age-specific, crude and age-standardized CFR Estimates for Germany

\begin{tabular}{|c|c|c|c|c|c|c|}
\hline Date & $\begin{array}{l}\text { Age } \\
\text { group }\end{array}$ & $\begin{array}{l}\text { Confirmed cases } \\
\mathrm{n}_{\mathrm{ij}}\end{array}$ & $\begin{array}{l}\text { Observed deaths } \\
\mathbf{d}_{\mathrm{ij}}\end{array}$ & $\begin{array}{l}\text { Age-specific crude CFR } \\
(\%)\end{array}$ & $\begin{array}{l}\text { European } \\
\text { Standard Population } \\
\text { (weights) }\end{array}$ & $\begin{array}{l}\text { Age-standardized CFR } \\
(\%)\end{array}$ \\
\hline \multirow[t]{8}{*}{13.05 .2020} & $0-9$ & 3,172 & 1 & 0.03 & 0.105 & \\
\hline & $10-19$ & 7,350 & 2 & 0.03 & 0.110 & \\
\hline & $20-49$ & 73,659 & 82 & 0.11 & 0.395 & \\
\hline & $50-69$ & 54,253 & 952 & 1.75 & 0.250 & \\
\hline & $70-89$ & 27,840 & 5,164 & 18.55 & 0.130 & \\
\hline & $90+$ & 4,898 & 1,428 & 29.15 & 0.010 & \\
\hline & Unknown & 134 & 5 & 3.73 & & \\
\hline & Total & 171,306 & 7,634 & 4.46 & 1.000 & 3.19 \\
\hline \multirow[t]{8}{*}{06.05 .2020} & $0-9$ & 2,931 & 1 & 0.03 & 0.105 & \\
\hline & $10-19$ & 6,959 & 1 & 0.01 & 0.110 & \\
\hline & $20-49$ & 70,671 & 72 & 0.10 & 0.395 & \\
\hline & $50-69$ & 52,636 & 860 & 1.63 & 0.250 & \\
\hline & $70-89$ & 26,746 & 4,750 & 17.76 & 0.130 & \\
\hline & $90+$ & 4,689 & 1,303 & 27.79 & 0.010 & \\
\hline & Unknown & 175 & 9 & 5.14 & & \\
\hline & Total & 164,807 & 6,996 & 4.24 & 1.000 & 3.04 \\
\hline \multirow[t]{8}{*}{ 29.04.2020 } & $0-9$ & 2,701 & 1 & 0.04 & 0.105 & \\
\hline & $10-19$ & 6,581 & 1 & 0.02 & 0.110 & \\
\hline & $20-49$ & 67,543 & 65 & 0.10 & 0.395 & \\
\hline & $50-69$ & 50,806 & 748 & 1.47 & 0.250 & \\
\hline & $70-89$ & 25,395 & 4,178 & 16.45 & 0.130 & \\
\hline & $90+$ & 4,420 & 1,116 & 25.25 & 0.010 & \\
\hline & Unknown & 195 & 6 & 3.08 & & \\
\hline & Total & 157,641 & 6,115 & 3.88 & 1.000 & 2.80 \\
\hline \multirow[t]{5}{*}{22.04 .2020} & $0-69$ & 118,545 & 657 & 0.55 & 0.860 & \\
\hline & $70-89$ & 23,069 & 3,351 & 14.53 & 0.130 & \\
\hline & $90+$ & 3,886 & 867 & 22.31 & 0.010 & \\
\hline & Unknown & 194 & 4 & 2.06 & & \\
\hline & Total & 145,694 & 4,879 & 3.35 & 1.000 & 2.59 \\
\hline \multirow[t]{5}{*}{15.04 .2020} & $0-59$ & 90,060 & 150 & 0.17 & 0.745 & \\
\hline & $60-79$ & 24,669 & 1,051 & 4.26 & 0.205 & \\
\hline & $80+$ & 12,625 & 2,048 & 16.22 & 0.050 & \\
\hline & Unknown & 230 & 5 & 2.17 & & \\
\hline & Total & 127,584 & 3,254 & 2.55 & 1.000 & 1.80 \\
\hline \multirow[t]{5}{*}{08.04 .2020} & $0-59$ & 74,617 & 87 & 0.12 & 0.745 & \\
\hline & $60-79$ & 19,935 & 620 & 3.11 & 0.205 & \\
\hline & $80+$ & 8,475 & 1,150 & 13.57 & 0.050 & \\
\hline & Unknown & 201 & 4 & 1.99 & & \\
\hline & Total & 103,228 & 1,861 & 1.80 & 1.000 & 1.41 \\
\hline
\end{tabular}




\begin{tabular}{|c|c|c|c|c|c|c|}
\hline Date & $\begin{array}{l}\text { Age } \\
\text { group }\end{array}$ & $\begin{array}{l}\text { Confirmed cases } \\
n_{i j}\end{array}$ & $\begin{array}{l}\text { Observed deaths } \\
\mathbf{d}_{\mathrm{ij}}\end{array}$ & $\begin{array}{l}\text { Age-specific crude CFR } \\
(\%)\end{array}$ & $\begin{array}{l}\text { European } \\
\text { Standard Population } \\
\text { (weights) }\end{array}$ & $\begin{array}{l}\text { Age-standardized CFR } \\
\text { (\%) }\end{array}$ \\
\hline \multirow[t]{5}{*}{ 01.04.2020 } & $0-59$ & 50,998 & 42 & 0.08 & 0.745 & \\
\hline & $60-79$ & 12,394 & 229 & 1.85 & 0.205 & \\
\hline & $80+$ & 3,784 & 459 & 12.13 & 0.050 & \\
\hline & Unknown & 190 & 2 & 1.05 & & \\
\hline & Total & 67,366 & 732 & 1.09 & 1.000 & 1.05 \\
\hline \multirow[t]{7}{*}{ 25.03.2020 } & $0-4$ & 234 & 0 & 0.00 & 0.050 & \\
\hline & $5-14$ & 651 & 0 & 0.00 & 0.110 & \\
\hline & $15-34$ & 7,877 & 0 & 0.00 & 0.240 & \\
\hline & $35-79$ & 21,507 & 48 & 0.22 & 0.550 & \\
\hline & $80+$ & 1,153 & 101 & 8.76 & 0.050 & \\
\hline & Unknown & 132 & 0 & 0.00 & & \\
\hline & Total & 31,554 & 149 & 0.47 & 1.000 & 0.56 \\
\hline \multirow[t]{7}{*}{ 18.03.2020 } & $0-4$ & 67 & 0 & 0.00 & 0.050 & \\
\hline & $5-14$ & 199 & 0 & 0.00 & 0.110 & \\
\hline & $15-34$ & 2,090 & 0 & 0.00 & 0.240 & \\
\hline & $35-59$ & 4,467 & 0 & 0.00 & 0.345 & \\
\hline & $60+$ & 1,337 & 12 & 0.90 & 0.255 & \\
\hline & Unknown & 38 & 0 & 0.00 & & \\
\hline & Total & 8,198 & 12 & 0.15 & 1.000 & 0.23 \\
\hline \multirow[t]{8}{*}{11.03 .2020} & $0-4$ & 15 & 0 & 0.00 & 0.050 & \\
\hline & $5-14$ & 28 & 0 & 0.00 & 0.110 & \\
\hline & $15-34$ & 340 & 0 & 0.00 & 0.240 & \\
\hline & $35-59$ & 551 & 0 & 0.00 & 0.345 & \\
\hline & $60-79$ & 128 & 2 & 1.56 & 0.205 & \\
\hline & $80+$ & 14 & 1 & 7.14 & 0.050 & \\
\hline & Unknown & 491 & 0 & 0.00 & & \\
\hline & Total & 1,567 & 3 & 0.19 & 1.000 & 0.68 \\
\hline \multirow[t]{8}{*}{ 04.03.2020 } & $0-4$ & 6 & 0 & 0.00 & 0.050 & \\
\hline & $5-14$ & 3 & 0 & 0.00 & 0.110 & \\
\hline & $15-34$ & 60 & 0 & 0.00 & 0.240 & \\
\hline & $35-59$ & 66 & 0 & 0.00 & 0.345 & \\
\hline & $60-79$ & 12 & 0 & 0.00 & 0.205 & \\
\hline & $80+$ & 0 & 0 & - & 0.050 & \\
\hline & Unknown & 115 & 0 & 0.00 & & \\
\hline & Total & 262 & 0 & 0.00 & 1.000 & 0.00 \\
\hline
\end{tabular}


Table 4

Age-specific, crude and age-standardized CFR Estimates for Italy

\begin{tabular}{|c|c|c|c|c|c|c|}
\hline Date & $\begin{array}{l}\text { Age } \\
\text { group }\end{array}$ & $\begin{array}{l}\text { Confirmed cases } \\
n_{i j}\end{array}$ & $\begin{array}{l}\text { Observed deaths } \\
d_{i j}\end{array}$ & $\begin{array}{l}\text { Age-specific crude CFR } \\
\text { (\%) }\end{array}$ & $\begin{array}{l}\text { European } \\
\text { Standard Population } \\
\text { (weights) }\end{array}$ & $\begin{array}{l}\text { Age-standardized CFR } \\
\text { (\%) }\end{array}$ \\
\hline \multirow[t]{12}{*}{14.05 .2020} & $0-9$ & 1,774 & 3 & 0.17 & 0.105 & \\
\hline & $10-19$ & 3,148 & 0 & 0.00 & 0.110 & \\
\hline & $20-29$ & 12,115 & 12 & 0.10 & 0.120 & \\
\hline & $30-39$ & 16,981 & 59 & 0.35 & 0.135 & \\
\hline & $40-49$ & 28,627 & 258 & 0.90 & 0.140 & \\
\hline & $50-59$ & 39,822 & 1,063 & 2.67 & 0.135 & \\
\hline & $60-69$ & 30,010 & 3,127 & 10.42 & 0.115 & \\
\hline & $70-79$ & 32,353 & 8,221 & 25.41 & 0.090 & \\
\hline & $80-89$ & 39,340 & 12,104 & 30.77 & 0.040 & \\
\hline & $90+$ & 17,852 & 4,844 & 27.13 & 0.010 & \\
\hline & Unknown & 52 & 1 & 1.92 & & \\
\hline & Total & 222,074 & 29,692 & 13.37 & 1.000 & 5.55 \\
\hline \multirow[t]{12}{*}{ 07.05.2020 } & $0-9$ & 1,642 & 3 & 0.18 & 0.105 & \\
\hline & $10-19$ & 2,908 & 0 & 0.00 & 0.110 & \\
\hline & $20-29$ & 11,457 & 9 & 0.08 & 0.120 & \\
\hline & $30-39$ & 16,189 & 54 & 0.33 & 0.135 & \\
\hline & $40-49$ & 27,553 & 246 & 0.89 & 0.140 & \\
\hline & $50-59$ & 38,399 & 993 & 2.59 & 0.135 & \\
\hline & $60-69$ & 29,252 & 2,976 & 10.2 & 0.115 & \\
\hline & $70-79$ & 31,627 & 7,849 & 24.82 & 0.090 & \\
\hline & $80-89$ & 38,042 & 11,395 & 29.95 & 0.040 & \\
\hline & $90+$ & 16,978 & 4,430 & 26.09 & 0.010 & \\
\hline & Unknown & 56 & 0 & 0.00 & & \\
\hline & Total & 214,103 & 27,955 & 13.06 & 1.000 & 5.41 \\
\hline \multirow[t]{12}{*}{ 28.04.2020 } & $0-9$ & 1,478 & 2 & 0.14 & 0.105 & \\
\hline & $10-19$ & 2,511 & 0 & 0.00 & 0.110 & \\
\hline & $20-29$ & 10,377 & 8 & 0.08 & 0.120 & \\
\hline & $30-39$ & 14,907 & 49 & 0.33 & 0.135 & \\
\hline & $40-49$ & 25,644 & 224 & 0.87 & 0.140 & \\
\hline & $50-59$ & 35,986 & 918 & 2.55 & 0.135 & \\
\hline & $60-69$ & 27,880 & 2,727 & 9.78 & 0.115 & \\
\hline & $70-79$ & 30,158 & 7,291 & 24.18 & 0.090 & \\
\hline & $80-89$ & 35,262 & 10,241 & 29.04 & 0.040 & \\
\hline & $90+$ & 15,186 & 3,755 & 24.73 & 0.010 & \\
\hline & Unknown & 81 & 0 & 0.00 & & \\
\hline & Total & 199,470 & 25,215 & 12.64 & 1.000 & 5.24 \\
\hline \multirow[t]{3}{*}{ 23.04.2020 } & $0-9$ & 1,304 & 2 & 0.15 & 0.105 & \\
\hline & $10-19$ & 2,146 & 0 & 0.00 & 0.110 & \\
\hline & $20-29$ & 8,963 & 7 & 0.08 & 0.120 & \\
\hline
\end{tabular}




\begin{tabular}{|c|c|c|c|c|c|c|}
\hline Date & $\begin{array}{l}\text { Age } \\
\text { group }\end{array}$ & $\begin{array}{l}\text { Confirmed cases } \\
\mathrm{n}_{\mathrm{ij}}\end{array}$ & $\begin{array}{l}\text { Observed deaths } \\
\mathbf{d}_{\mathrm{ij}}\end{array}$ & $\begin{array}{l}\text { Age-specific crude CFR } \\
(\%)\end{array}$ & $\begin{array}{l}\text { European } \\
\text { Standard Population } \\
\text { (weights) }\end{array}$ & $\begin{array}{l}\text { Age-standardized CFR } \\
(\%)\end{array}$ \\
\hline & $30-39$ & 13,137 & 48 & 0.37 & 0.135 & \\
\hline & $40-49$ & 22,767 & 203 & 0.89 & 0.140 & \\
\hline & $50-59$ & 32,524 & 861 & 2.65 & 0.135 & \\
\hline & $60-69$ & 25,707 & 2,576 & 10.02 & 0.115 & \\
\hline & $70-79$ & 27,615 & 6,882 & 24.92 & 0.090 & \\
\hline & $80-89$ & 30,534 & 9,396 & 30.77 & 0.040 & \\
\hline & $90+$ & 12,328 & 3,213 & 26.06 & 0.010 & \\
\hline & Unknown & 118 & 0 & 0.00 & & \\
\hline & Total & 177,143 & 23,188 & 13.09 & 1.000 & 5.44 \\
\hline \multirow[t]{12}{*}{16.04 .2020} & $0-9$ & 1,123 & 1 & 0.09 & 0.105 & \\
\hline & $10-19$ & 1,804 & 0 & 0.00 & 0.110 & \\
\hline & $20-29$ & 7,737 & 7 & 0.09 & 0.120 & \\
\hline & $30-39$ & 11,686 & 40 & 0.34 & 0.135 & \\
\hline & $40-49$ & 20,519 & 178 & 0.87 & 0.140 & \\
\hline & $50-59$ & 29,858 & 756 & 2.53 & 0.135 & \\
\hline & $60-69$ & 24,040 & 2,284 & 9.50 & 0.115 & \\
\hline & $70-79$ & 25,717 & 6,203 & 24.12 & 0.090 & \\
\hline & $80-89$ & 26,706 & 8,070 & 30.22 & 0.040 & \\
\hline & $90+$ & 9,813 & 2,455 & 25.02 & 0.010 & \\
\hline & Unknown & 104 & 2 & 1.92 & & \\
\hline & Total & 159,107 & 19,996 & 12.57 & 1.000 & 5.25 \\
\hline \multirow[t]{12}{*}{09.04 .2020} & $0-9$ & 938 & 1 & 0.11 & 0.105 & \\
\hline & $10-19$ & 1,432 & 0 & 0.00 & 0.110 & \\
\hline & $20-29$ & 6,360 & 7 & 0.11 & 0.120 & \\
\hline & $30-39$ & 9,956 & 36 & 0.36 & 0.135 & \\
\hline & $40-49$ & 17,745 & 153 & 0.86 & 0.140 & \\
\hline & $50-59$ & 26,391 & 638 & 2.42 & 0.135 & \\
\hline & $60-69$ & 21,734 & 1,957 & 9.00 & 0.115 & \\
\hline & $70-79$ & 22,934 & 5,366 & 23.40 & 0.090 & \\
\hline & $80-89$ & 21,636 & 6,711 & 31.02 & 0.040 & \\
\hline & $90+$ & 6,842 & 1,784 & 26.07 & 0.010 & \\
\hline & Unknown & 142 & 1 & 0.70 & & \\
\hline & Total & 136,110 & 16,654 & 12.24 & 1.000 & 5.16 \\
\hline \multirow[t]{7}{*}{ 02.04.2020 } & $0-9$ & 693 & 0 & 0.00 & 0.105 & \\
\hline & $10-19$ & 931 & 0 & 0.00 & 0.110 & \\
\hline & $20-29$ & 4,530 & 6 & 0.13 & 0.120 & \\
\hline & $30-39$ & 7,466 & 29 & 0.39 & 0.135 & \\
\hline & $40-49$ & 13,701 & 110 & 0.80 & 0.140 & \\
\hline & $50-59$ & 20,975 & 479 & 2.28 & 0.135 & \\
\hline & $60-69$ & 18,089 & 1,448 & 8.00 & 0.115 & \\
\hline
\end{tabular}




\begin{tabular}{|c|c|c|c|c|c|c|}
\hline \multirow[t]{6}{*}{ Date } & $\begin{array}{l}\text { Age } \\
\text { group }\end{array}$ & $\begin{array}{l}\text { Confirmed cases } \\
\mathrm{n}_{\mathrm{ij}}\end{array}$ & $\begin{array}{l}\text { Observed deaths } \\
d_{i j}\end{array}$ & $\begin{array}{l}\text { Age-specific crude CFR } \\
\text { (\%) }\end{array}$ & $\begin{array}{l}\text { European } \\
\text { Standard Population } \\
\text { (weights) }\end{array}$ & $\begin{array}{l}\text { Age-standardized CFR } \\
\text { (\%) }\end{array}$ \\
\hline & $70-79$ & 19,238 & 4,196 & 21.81 & 0.090 & \\
\hline & $80-89$ & 16,252 & 5,029 & 30.94 & 0.040 & \\
\hline & $90+$ & 4,356 & 1,251 & 28.72 & 0.010 & \\
\hline & Unknown & 168 & 2 & 1.19 & & \\
\hline & Total & 106,399 & 12,550 & 11.80 & 1.000 & 4.90 \\
\hline \multirow[t]{12}{*}{ 26.03.2020 } & $0-9$ & 428 & 0 & 0.00 & 0.105 & \\
\hline & $10-19$ & 512 & 0 & 0.00 & 0.110 & \\
\hline & $20-29$ & 2,778 & 0 & 0.00 & 0.120 & \\
\hline & $30-39$ & 5,033 & 17 & 0.34 & 0.135 & \\
\hline & $40-49$ & 9,295 & 67 & 0.72 & 0.140 & \\
\hline & $50-59$ & 14,508 & 243 & 1.67 & 0.135 & \\
\hline & $60-69$ & 13,243 & 761 & 5.75 & 0.115 & \\
\hline & $70-79$ & 14,198 & 2,403 & 16.92 & 0.090 & \\
\hline & $80-89$ & 11,001 & 2,702 & 24.56 & 0.040 & \\
\hline & $90+$ & 2,538 & 608 & 23.96 & 0.010 & \\
\hline & Unknown & 246 & 0 & 0.00 & & \\
\hline & Total & 73,780 & 6,801 & 9.22 & 1.000 & 3.78 \\
\hline \multirow[t]{12}{*}{ 19.03.2020 } & $0-9$ & 205 & 0 & 0.00 & 0.105 & \\
\hline & $10-19$ & 270 & 0 & 0.00 & 0.110 & \\
\hline & $20-29$ & 1,374 & 0 & 0.00 & 0.120 & \\
\hline & $30-39$ & 2,525 & 9 & 0.36 & 0.135 & \\
\hline & $40-49$ & 4,396 & 25 & 0.57 & 0.140 & \\
\hline & $50-59$ & 6,834 & 83 & 1.21 & 0.135 & \\
\hline & $60-69$ & 6,337 & 312 & 4.92 & 0.115 & \\
\hline & $70-79$ & 7,121 & 1,090 & 15.31 & 0.090 & \\
\hline & $80-89$ & 5,352 & 1,243 & 23.22 & 0.040 & \\
\hline & $90+$ & 1,115 & 285 & 25.56 & 0.010 & \\
\hline & Unknown & 202 & 0 & 0.00 & & \\
\hline & Total & 35,731 & 3,047 & 8.53 & 1.000 & 3.42 \\
\hline \multirow[t]{7}{*}{11.03 .2020} & $0-49$ & 2,484 & 1 & 0.04 & 0.610 & \\
\hline & $50-59$ & 2,000 & 10 & 0.50 & 0.135 & \\
\hline & $60-69$ & 2,080 & 52 & 2.50 & 0.115 & \\
\hline & $70-79$ & 2,477 & 213 & 8.60 & 0.090 & \\
\hline & $80+$ & 2,142 & 332 & 15.50 & 0.050 & \\
\hline & Unknown & 355 & 11 & 3.10 & & \\
\hline & Total & 11,538 & 619 & 5.36 & 1.000 & 1.93 \\
\hline \multirow[t]{4}{*}{ 04.03.2020 } & $0-69$ & 1,600 & 8 & 0.50 & 0.860 & \\
\hline & $70-79$ & 453 & 24 & 5.30 & 0.090 & \\
\hline & $80+$ & 376 & 41 & 10.90 & 0.050 & \\
\hline & Unknown & 571 & 32 & 5.60 & & \\
\hline
\end{tabular}




\begin{tabular}{|c|c|c|c|c|c|c|}
\hline Date & $\begin{array}{l}\text { Age } \\
\text { group }\end{array}$ & $\begin{array}{l}\text { Confirmed cases } \\
\mathrm{n}_{\mathrm{ij}}\end{array}$ & $\begin{array}{l}\text { Observed deaths } \\
d_{i j}\end{array}$ & $\begin{array}{l}\text { Age-specific crude CFR } \\
(\%)\end{array}$ & $\begin{array}{l}\text { European } \\
\text { Standard Population } \\
\text { (weights) }\end{array}$ & $\begin{array}{l}\text { Age-standardized CFF } \\
\text { (\%) }\end{array}$ \\
\hline & Total & 3,000 & 105 & 3.50 & 1.000 & 1.45 \\
\hline
\end{tabular}


Table 5

Age-specific, crude and age-standardized CFR Estimates for France

\begin{tabular}{|c|c|c|c|c|c|c|}
\hline Date & $\begin{array}{l}\text { Age } \\
\text { group }\end{array}$ & Confirmed cases $n_{i j}$ & Observed deaths $d_{i j}$ & Age-specific crude CFR (\%) & $\begin{array}{l}\text { European } \\
\text { Standard } \\
\text { Population (weights) }\end{array}$ & Age-standardized CFR (\%) \\
\hline \multirow[t]{7}{*}{12.05 .2020} & $0-14$ & 769 & 3 & 0.39 & 0.160 & \\
\hline & $15-44$ & 9,911 & 163 & 1.64 & 0.380 & \\
\hline & $45-64$ & 24,342 & 1,717 & 7.05 & 0.265 & \\
\hline & $65-74$ & 18,446 & 2,998 & 16.25 & 0.105 & \\
\hline & $75+$ & 42,246 & 12,025 & 28.46 & 0.090 & \\
\hline & Unknown & 44,513 & 10,085 & 22.66 & & \\
\hline & Total & 140,227 & 26,991 & 19.25 & 1.000 & 6.82 \\
\hline \multirow[t]{7}{*}{05.05 .2020} & $0-14$ & 792 & 2 & 0.25 & 0.160 & \\
\hline & $15-44$ & 9,610 & 153 & 1.59 & 0.380 & \\
\hline & $45-64$ & 23,849 & 1,591 & 6.67 & 0.265 & \\
\hline & $65-74$ & 18,019 & 2,821 & 15.66 & 0.105 & \\
\hline & $75+$ & 40,643 & 11,396 & 28.04 & 0.090 & \\
\hline & Unknown & 40,054 & 9,598 & 23.96 & & \\
\hline & Total & 132,967 & 25,561 & 19.22 & 1.000 & 6.58 \\
\hline \multirow[t]{7}{*}{ 28.04.2020 } & $0-14$ & 740 & 2 & 0.27 & 0.160 & \\
\hline & $15-44$ & 9,205 & 137 & 1.49 & 0.380 & \\
\hline & $45-64$ & 23,077 & 1,474 & 6.39 & 0.265 & \\
\hline & $65-74$ & 17,336 & 2,586 & 14.92 & 0.105 & \\
\hline & $75+$ & 38,179 & 10,519 & 27.55 & 0.090 & \\
\hline & Unknown & 38,298 & 8,968 & 23.42 & & \\
\hline & Total & 126,835 & 23,686 & 18.67 & 1.000 & 6.35 \\
\hline \multirow[t]{7}{*}{21.04 .2020} & $0-14$ & 671 & 2 & 0.30 & 0.160 & \\
\hline & $15-44$ & 8,590 & 125 & 1.46 & 0.380 & \\
\hline & $45-64$ & 21,828 & 1,292 & 5.92 & 0.265 & \\
\hline & $65-74$ & 16,182 & 2,248 & 13.89 & 0.105 & \\
\hline & $75+$ & 34,301 & 9,153 & 26.68 & 0.090 & \\
\hline & Unknown & 35,752 & 7,976 & 22.31 & & \\
\hline & Total & 117,324 & 20,796 & 17.73 & 1.000 & 6.03 \\
\hline \multirow[t]{7}{*}{ 14.04.2020 } & $0-14$ & 592 & 1 & 0.17 & 0.160 & \\
\hline & $15-44$ & 7,524 & 105 & 1.40 & 0.380 & \\
\hline & $45-64$ & 19,689 & 1,016 & 5.16 & 0.265 & \\
\hline & $65-74$ & 14,405 & 1,769 & 12.28 & 0.105 & \\
\hline & $75+$ & 28,438 & 7,171 & 25.22 & 0.090 & \\
\hline & Unknown & 32,925 & 6,591 & 20.02 & & \\
\hline & Total & 103,573 & 16,653 & 16.08 & 1.000 & 5.48 \\
\hline \multirow[t]{3}{*}{07.04 .2020} & $0-14$ & 451 & 0 & 0.00 & 0.160 & \\
\hline & $15-44$ & 6,242 & 65 & 1.04 & 0.380 & \\
\hline & $45-64$ & 16,448 & 736 & 4.47 & 0.265 & \\
\hline
\end{tabular}




\begin{tabular}{|c|c|c|c|c|c|c|}
\hline Date & $\begin{array}{l}\text { Age } \\
\text { group }\end{array}$ & Confirmed cases $n_{i j}$ & Observed deaths $d_{i j}$ & Age-specific crude CFR (\%) & $\begin{array}{l}\text { European } \\
\text { Standard } \\
\text { Population (weights) }\end{array}$ & Age-standardized CFR (\%) \\
\hline & $65-74$ & 11,924 & 1,257 & 10.54 & 0.105 & \\
\hline & $75+$ & 20,826 & 4,975 & 23.89 & 0.090 & \\
\hline & Unknown & 38,919 & 3,295 & 8.47 & & \\
\hline & Total & 94,810 & 10,328 & 10.89 & 1.000 & 4.84 \\
\hline \multirow[t]{7}{*}{31.03 .2020} & $0-14$ & 309 & 0 & 0.00 & 0.160 & \\
\hline & $15-44$ & 4,168 & 29 & 0.70 & 0.380 & \\
\hline & $45-64$ & 10,525 & 319 & 3.03 & 0.265 & \\
\hline & $65-74$ & 7,677 & 589 & 7.67 & 0.105 & \\
\hline & $75+$ & 12,524 & 2,539 & 20.27 & 0.090 & \\
\hline & Unknown & 168 & 2 & 1.19 & & \\
\hline & Total & 35,371 & 3,478 & 9.83 & 1.000 & 3.70 \\
\hline \multirow[t]{7}{*}{ 24.03.2020 } & $0-14$ & 167 & 0 & 0.00 & 0.160 & \\
\hline & $15-44$ & 3,882 & 5 & 0.13 & 0.380 & \\
\hline & $45-64$ & 4,204 & 32 & 0.76 & 0.265 & \\
\hline & $65-74$ & 1,778 & 73 & 4.11 & 0.105 & \\
\hline & $75+$ & 2,675 & 397 & 14.84 & 0.090 & \\
\hline & Unknown & 9,596 & 593 & 6.18 & & \\
\hline & Total & 22,302 & 1,100 & 4.93 & 1.000 & 2.02 \\
\hline \multirow[t]{7}{*}{15.03 .2020} & $0-14$ & 126 & 0 & 0.00 & 0.160 & \\
\hline & $15-44$ & 1,808 & 2 & 0.11 & 0.380 & \\
\hline & $45-64$ & 2,067 & 11 & 0.53 & 0.265 & \\
\hline & $65-74$ & 850 & 20 & 2.35 & 0.105 & \\
\hline & $75+$ & 1,236 & 127 & 10.28 & 0.090 & \\
\hline & Unknown & 291 & 1 & 0.34 & & \\
\hline & Total & 6,378 & 161 & 2.52 & 1.000 & 1.35 \\
\hline \multirow[t]{6}{*}{10.03 .2020} & $0-17$ & 87 & 1 & 1.15 & 0.193 & \\
\hline & $18-64$ & 1,111 & 6 & 0.54 & 0.612 & \\
\hline & $65-74$ & 272 & 5 & 1.84 & 0.105 & \\
\hline & $75+$ & 349 & 33 & 9.46 & 0.090 & \\
\hline & Unknown & 220 & 0 & 0.00 & & \\
\hline & Total & 2,039 & 45 & 2.21 & 1.000 & 1.60 \\
\hline \multirow[t]{5}{*}{04.03 .2020} & $0-17$ & 60 & NA & - & 0.193 & \\
\hline & $18-64$ & 148 & NA & - & 0.612 & \\
\hline & $65-74$ & 31 & NA & - & 0.105 & \\
\hline & $75+$ & 46 & NA & - & 0.090 & \\
\hline & Total & 285 & 4 & 1.40 & 1.000 & NA \\
\hline
\end{tabular}


Table 6

Age-specific, crude and age-standardized CFR Estimates for Spain

\begin{tabular}{|c|c|c|c|c|c|c|}
\hline Date & $\begin{array}{l}\text { Age } \\
\text { group }\end{array}$ & Confirmed cases $n_{i j}$ & Observed deaths $d_{i j}$ & Age-specific crude CFR (\%) & $\begin{array}{l}\text { European } \\
\text { Standard } \\
\text { Population (weights) }\end{array}$ & Age-standardized CFR (\%) \\
\hline \multirow[t]{12}{*}{11.05 .2020} & $0-2$ & 381 & 2 & 0.52 & 0.030 & \\
\hline & $2-4$ & 192 & 0 & 0.00 & 0.010 & \\
\hline & $5-14$ & 826 & 1 & 0.12 & 0.110 & \\
\hline & $15-29$ & 15,421 & 28 & 0.18 & 0.170 & \\
\hline & $30-39$ & 23,489 & 63 & 0.27 & 0.135 & \\
\hline & $40-49$ & 36,042 & 217 & 0.60 & 0.135 & \\
\hline & $50-59$ & 43,668 & 653 & 1.50 & 0.130 & \\
\hline & $60-69$ & 35,074 & 1,820 & 5.19 & 0.110 & \\
\hline & $70-79$ & 33,345 & 4,884 & 14.65 & 0.110 & \\
\hline & $80+$ & 58,731 & 12,839 & 21.86 & 0.060 & \\
\hline & Unknown & 3,118 & 6,413 & 205.68 & & \\
\hline & Total & 250,287 & 26,920 & 10.76 & 1.000 & 3.87 \\
\hline \multirow[t]{12}{*}{06.05 .2020} & $0-2$ & 331 & 2 & 0.60 & 0.030 & \\
\hline & $2-4$ & 158 & 0 & 0.00 & 0.010 & \\
\hline & $5-14$ & 633 & 0 & 0.00 & 0.110 & \\
\hline & $15-29$ & 13,054 & 26 & 0.20 & 0.170 & \\
\hline & $30-39$ & 20,406 & 55 & 0.27 & 0.135 & \\
\hline & $40-49$ & 31,556 & 185 & 0.59 & 0.135 & \\
\hline & $50-59$ & 38,540 & 560 & 1.45 & 0.130 & \\
\hline & $60-69$ & 31,610 & 1,524 & 4.82 & 0.110 & \\
\hline & $70-79$ & 30,312 & 4,273 & 14.10 & 0.110 & \\
\hline & $80+$ & 49,268 & 10,619 & 21.55 & 0.060 & \\
\hline & Unknown & 5,579 & 8,613 & 154.38 & & \\
\hline & Total & 221,447 & 26,070 & 11.77 & 1.000 & 3.73 \\
\hline \multirow[t]{12}{*}{ 29.04.2020 } & $0-2$ & 309 & 2 & 0.65 & 0.030 & \\
\hline & $2-4$ & 138 & 0 & 0.00 & 0.010 & \\
\hline & $5-14$ & 537 & 0 & 0.00 & 0.110 & \\
\hline & $15-29$ & 11,893 & 25 & 0.21 & 0.170 & \\
\hline & $30-39$ & 18,825 & 53 & 0.28 & 0.135 & \\
\hline & $40-49$ & 29,350 & 172 & 0.59 & 0.135 & \\
\hline & $50-59$ & 36,126 & 497 & 1.38 & 0.130 & \\
\hline & $60-69$ & 30,112 & 1,392 & 4.62 & 0.110 & \\
\hline & $70-79$ & 29,270 & 3,996 & 13.65 & 0.110 & \\
\hline & $80+$ & 46,361 & 9,727 & 20.98 & 0.060 & \\
\hline & Unknown & 10,514 & 8,679 & 82.55 & & \\
\hline & Total & 213,435 & 24,543 & 11.50 & 1.000 & 3.62 \\
\hline \multirow[t]{2}{*}{22.04 .2020} & $0-2$ & 238 & 2 & 0.84 & 0.030 & \\
\hline & $2-4$ & 95 & 0 & 0.00 & 0.010 & \\
\hline
\end{tabular}




\begin{tabular}{|c|c|c|c|c|c|c|}
\hline Date & $\begin{array}{l}\text { Age } \\
\text { group }\end{array}$ & Confirmed cases $n_{i j}$ & Observed deaths $d_{i j}$ & Age-specific crude CFR (\%) & $\begin{array}{l}\text { European } \\
\text { Standard } \\
\text { Population (weights) }\end{array}$ & Age-standardized CFR (\%) \\
\hline & $5-14$ & 406 & 0 & 0.00 & 0.110 & \\
\hline & $15-29$ & 8,223 & 23 & 0.28 & 0.170 & \\
\hline & $30-39$ & 13,819 & 40 & 0.29 & 0.135 & \\
\hline & $40-49$ & 21,611 & 134 & 0.62 & 0.135 & \\
\hline & $50-59$ & 26,898 & 393 & 1.46 & 0.130 & \\
\hline & $60-69$ & 23,022 & 1,114 & 4.84 & 0.110 & \\
\hline & $70-79$ & 22,013 & 3,291 & 14.95 & 0.110 & \\
\hline & $80+$ & 31,155 & 7,635 & 24.51 & 0.060 & \\
\hline & Unknown & 65,544 & 9,525 & 14.53 & & \\
\hline & Total & 213,024 & 22,157 & 10.40 & 1.000 & 4.03 \\
\hline \multirow[t]{12}{*}{15.04 .2020} & $0-2$ & 171 & 2 & 1.17 & 0.030 & \\
\hline & $2-4$ & 69 & 0 & 0.00 & 0.010 & \\
\hline & $5-14$ & 325 & 0 & 0.00 & 0.110 & \\
\hline & $15-29$ & 6,761 & 17 & 0.25 & 0.170 & \\
\hline & $30-39$ & 11,662 & 33 & 0.28 & 0.135 & \\
\hline & $40-49$ & 18,259 & 110 & 0.60 & 0.135 & \\
\hline & $50-59$ & 22,788 & 298 & 1.31 & 0.130 & \\
\hline & $60-69$ & 20,022 & 886 & 4.43 & 0.110 & \\
\hline & $70-79$ & 18,997 & 2,650 & 13.95 & 0.110 & \\
\hline & $80+$ & 22,973 & 5,638 & 24.54 & 0.060 & \\
\hline & Unknown & 60,789 & 9,681 & 15.93 & & \\
\hline & Total & 182,816 & 19,315 & 10.57 & 1.000 & 3.86 \\
\hline \multirow[t]{12}{*}{05.04 .2020} & $0-2$ & 98 & 1 & 1.02 & 0.030 & \\
\hline & $2-4$ & 47 & 0 & 0.00 & 0.010 & \\
\hline & $5-14$ & 230 & 0 & 0.00 & 0.110 & \\
\hline & $15-29$ & 4,668 & 6 & 0.13 & 0.170 & \\
\hline & $30-39$ & 8,348 & 14 & 0.17 & 0.135 & \\
\hline & $40-49$ & 12,733 & 46 & 0.36 & 0.135 & \\
\hline & $50-59$ & 15,754 & 125 & 0.79 & 0.130 & \\
\hline & $60-69$ & 14,021 & 378 & 2.70 & 0.110 & \\
\hline & $70-79$ & 13,446 & 1,201 & 8.93 & 0.110 & \\
\hline & $80+$ & 13,810 & 2,636 & 19.09 & 0.060 & \\
\hline & Unknown & 51,346 & 8,934 & 17.40 & & \\
\hline & Total & 134,501 & 13,341 & 9.92 & 1.000 & 2.65 \\
\hline \multirow[t]{6}{*}{31.03 .2020} & $0-2$ & 98 & 1 & 1.02 & 0.030 & \\
\hline & $2-4$ & 39 & 0 & 0.00 & 0.010 & \\
\hline & $5-14$ & 176 & 0 & 0.00 & 0.110 & \\
\hline & $15-29$ & 3,048 & 6 & 0.20 & 0.170 & \\
\hline & $30-39$ & 5,335 & 11 & 0.21 & 0.135 & \\
\hline & $40-49$ & 7,960 & 41 & 0.52 & 0.135 & \\
\hline
\end{tabular}




\begin{tabular}{|c|c|c|c|c|c|c|}
\hline \multirow[t]{7}{*}{ Date } & $\begin{array}{l}\text { Age } \\
\text { group }\end{array}$ & Confirmed cases $n_{i j}$ & Observed deaths $d_{i j}$ & Age-specific crude CFR (\%) & $\begin{array}{l}\text { European } \\
\text { Standard } \\
\text { Population (weights) }\end{array}$ & Age-standardized CFR (\%) \\
\hline & $50-59$ & 9,449 & 86 & 0.91 & 0.130 & \\
\hline & $60-69$ & 8,694 & 272 & 3.13 & 0.110 & \\
\hline & $70-79$ & 8,648 & 857 & 9.91 & 0.110 & \\
\hline & $80+$ & 8,322 & 1,802 & 21.65 & 0.060 & \\
\hline & Unknown & 50,367 & 6,311 & 12.53 & & \\
\hline & Total & 102,136 & 9,387 & 9.19 & 1.000 & 3.01 \\
\hline \multirow[t]{12}{*}{ 24.03.2020 } & $0-2$ & 60 & 0 & 0.00 & 0.030 & \\
\hline & $2-4$ & 20 & 0 & 0.00 & 0.010 & \\
\hline & $5-14$ & 112 & 0 & 0.00 & 0.110 & \\
\hline & $15-29$ & 1,513 & 3 & 0.20 & 0.170 & \\
\hline & $30-39$ & 2,349 & 3 & 0.13 & 0.135 & \\
\hline & $40-49$ & 3,122 & 8 & 0.26 & 0.135 & \\
\hline & $50-59$ & 3,416 & 12 & 0.35 & 0.130 & \\
\hline & $60-69$ & 3,162 & 58 & 1.83 & 0.110 & \\
\hline & $70-79$ & 3,339 & 151 & 4.52 & 0.110 & \\
\hline & $80+$ & 3,248 & 486 & 14.96 & 0.060 & \\
\hline & Unknown & 26,962 & 2,926 & 10.85 & & \\
\hline & Total & 47,303 & 3,647 & 7.71 & 1.000 & 1.73 \\
\hline \multirow[t]{8}{*}{18.03 .2020} & $0-1$ & 3 & 0 & 0.00 & 0.010 & \\
\hline & $1-4$ & 5 & 0 & 0.00 & 0.030 & \\
\hline & $5-14$ & 23 & 0 & 0.00 & 0.110 & \\
\hline & $15-44$ & 432 & 0 & 0.00 & 0.380 & \\
\hline & $45-64$ & 413 & 0 & 0.00 & 0.270 & \\
\hline & $65+$ & 381 & 0 & 0.00 & 0.200 & \\
\hline & Unknown & 12,459 & 623 & 0.05 & & \\
\hline & Total & 13,716 & 623 & 0.05 & 1.000 & 0.00 \\
\hline \multirow[t]{8}{*}{11.03 .2020} & $0-1$ & 0 & 0 & - & 0.010 & \\
\hline & $1-4$ & 1 & 0 & 0.00 & 0.030 & \\
\hline & $5-14$ & 2 & 0 & 0.00 & 0.110 & \\
\hline & $15-44$ & 87 & 0 & 0.00 & 0.380 & \\
\hline & $45-64$ & 81 & 0 & 0.00 & 0.270 & \\
\hline & $65+$ & 57 & 0 & 0.00 & 0.200 & \\
\hline & Unknown & 1,900 & 54 & 0.03 & & \\
\hline & Total & 2,128 & 54 & 0.03 & 1.000 & 0.00 \\
\hline \multirow[t]{6}{*}{04.03 .2020} & $0-14$ & 0 & 0 & - & 0.150 & \\
\hline & $15-44$ & 48 & 0 & 0.00 & 0.380 & \\
\hline & $45-64$ & 38 & 0 & 0.00 & 0.270 & \\
\hline & $65+$ & 21 & 0 & 0.00 & 0.200 & \\
\hline & Unknown & 144 & 3 & 0.01 & & \\
\hline & Total & 251 & 3 & 1.20 & 1.000 & 0.00 \\
\hline
\end{tabular}


Aim 2. Assess different potential time lags for the CFR estimates

Figure 3 shows the crude estimates for the CFR during the study period using lags of 0 to 10 days.

Figure 3. Crude CFR estimates for Germany, Italy, France and Spain from March 4th, 2020 until May 14th, 2020, with different time lags Sources: (43); Own computation and design

For all countries, the estimates with different lags converged, since the number of newly detected cases was strongly decreasing towards the end of our observation period. In Germany and France, a longer time lag, such as 8 to 10 days, yielded estimates over time, which were close to the current estimates. A lag of about 4 days yielded fairly constant estimates over time in Italy and Spain. The most recent estimates for May 14 th, 2020 yielded crude CFR estimates ranging between lags of 0 to 10 days of 4.5-4.7, 14.0-14.8, 19.3-20.6, 11.9-12.5 for Germany, Italy, France, and Spain, respectively. Ignoring the time lag yielded to an underestimation of the CFR at peak times of the pandemic. This underestimation was stronger in Germany, and therefore the comparison of the estimates with those from the other three countries showed larger differences at this period compared to mid-April. For example, the estimated crude CFRs in Germany and Spain on March 21st were $0.24 \%$ and $5 \%$, respectively. On April 16th, these were $2.7 \%$ and $10.4 \%$ and on May 14 th the numbers were $4.5 \%$ and $11.9 \%$. The corresponding CFR ratios decreased from 20.8 to 3.9 to 2.6 .

Aim 3. Describe the association between case fatality and the intensive care bed capacity

We define the daily hospitalization fatality as the deaths on a particular day relative to the total number of hospitalized cases admitted during the previous two weeks. In Italy, e.g., 195 persons died on May 14th, 2020, and 10,341 cases were hospitalized in the previous two weeks, which leads to a daily hospitalization fatality of $1.89 \%$. Figure 4 illustrates the daily fatality of hospitalized cases in association with the needed intensive care bed capacities. The total available intensive care bed capacities, taken from OECD (44), are indicated by vertical lines.

Figure 4. Daily hospitalization fatality relative to intensive care beds needed for COVID-19 patients Sources: (43-48); Own computation and design

The countries in our analysis were at different stages in terms of the capacity utilization of their intensive care beds. At the end of our analysis, Italy had the highest number of COVID-19 cases requiring a treatment in an intensive care unit $(1,722)$, followed by France $(1,596)$, Spain $(1,174)$, and Germany $(690)$. We observe a positive correlation of the daily hospitalization fatalities with the intensive care beds needed for COVID-19 patients on a particular day in France, Italy, and Spain. For Germany, the number of intensive care beds occupied by COVID-19 patients has so far consistently been below the available capacity.

\section{Discussion}

There are large differences in the reported CFRs between countries. We discussed factors that may explain shares of these differences. We present evidence that a large proportion of the differences of CFRs between the four countries included here can be attributed to different age distributions of cases. Moreover, we have shown, that crude CFR estimates are strongly biased at peak phases of the pandemic due to lags between case reporting and death. This bias becomes smaller when daily case numbers decrease. It is unclear, how different CFRs among the European countries are indeed an indicator of differences in healthcare capacity. In the countries considered here, we could show an association between the number of intensive care beds needed for COVID-19 patients and daily hospitalization fatality.

Our analysis only considered the impact of the three factors demographics, delay in deaths after infection, and healthcare system capacity on CFR estimates of COVID-19. There are certainly other factors, which play into country-specific differences as well. Among those may be environmental factors, such as air pollution or climatic circumstances (52). Moreover, there are differences in the overall mortality among countries (53), which should be taken into account for a holistic international comparison of COVID-19 and general cause-specific mortality.

Generally, differences in CFR estimates which remain after adjusting for population structure, time lags, and health care capacity are most likely an indicator of different extents of underreporting of COVID-19 cases across countries caused by limited surveillance capacities of the countries' health systems. Compared to crude CFRs in the countries assessed, our findings of less diverse hospitalization fatality suggest that varying underreporting of cases may be responsible for a substantial part of the difference in estimated age-standardized CFRs. Underassessment of COVID-19 cases can be assumed for most countries, with varying extent (54). E.g., a recent seroprevalence studies assessing underassessment of reported cases from Geneva, Switzerland estimates that only one of 11.6 cases were actually reported in April and May in Geneva (55).

For persons not belonging to population groups at high risk of severe COVID-19 clinical courses, the disease in some cases appears with only minor symptoms or no symptoms at all (56). Thus, many mild cases may not appear in the statistics and therefore bias the data towards the more severe cases (2, 19), overestimating the overall fatality risk. For example, the number of undocumented cases was estimated to be $86 \%$ of all infections in China at the end of January (15) and $72 \%$ of all infections in Italy using international travel data at the end of February (57). One example of a situation with presumably very low underassessment is a study conducted on the cruise ship Diamond Princess (58). All passengers were tested, and 696 Passengers were tested positive. According to recent data, thirteen individuals have died due to COVID-19, yielding a crude fatality risk of $1.83 \%$ (59), resulting from the passengers' high mean age. The age-standardized CFR in this population is $0.65 \%$. Although the study population was small yielding an imprecise estimate, it may roughly indicate the proportion of unobserved cases among other populations, assuming the fatality in the ship's population was comparable to that of the countries considered in this paper.

Besides underassessment due to underdetection of asymptomatic or mildly symptomatic cases, the diagnostic accuracy of tests plays a role. The WHO recommends RT-PCR to detect the virus in pharyngeal swabs (18). Differences in viral load at various time points after infection and at different locations (16) 
make a reliable detection of the virus difficult. A study of Fang et al. showed $71 \%$ sensitivity of RT-PCR compared to $98 \%$ of chest-CT (13). Thus, low sensitivity would also result in underreporting of COVID-19 and therefore in an overestimation of case fatality.

The extent of underassessment of cases can also be assumed to vary during country infection dynamics. Underreporting may appear more severely in the early and later stages of the epidemic (2), as the disease in the earlier stages can be mistaken as another respiratory syndrome due to similar symptoms (60). In the peak stages of the epidemic, there might be an overload of the reporting system due to the high number of cases and limited capacities of laboratories (18), which might lead to a general underestimation of cases and therefore an overestimation of the fatality risks.

As an important limitation of our work, we found that public data on the age structure of infected and deceased were missing in public reports on COVID-19 in many European countries. Even for the included countries, this data was partly only available at specific time points, for roughly aggregated age groups, or only for a selection of all reported cases or deaths. For other countries, age-specific data are not openly available at all. Another limitation of this work is that we were not able to gain information on the distribution of comorbidities relevant to COVID-19 over age groups of infected and deceased in the European countries assessed, thus limiting our understanding of differences in CFR estimates due to differences in comorbidities. For the analysis of the association between fatality and the healthcare load measured by intensive care beds needed, we could not incorporate the age structure or severity of hospitalized cases into our computations, because these data were not available. Additionally, we did not have access to daily numbers of intensive care beds available for COVID-19 patients, which is why we chose to retain the absolute values of intensive care beds needed.

For publicly available data to have public health consequences, better reporting of data on healthcare capacities on a daily or at least weekly scale is needed in Europe. More detailed data on the demographics of the cases and deaths would help our understanding of the demographic impact on the CFRs. There are very few countries providing this information in a sufficiently detailed form (such as Spain and Italy do) and many countries do not offer age-stratified data at all or do not provide their data by sex. This biases our understanding of the severity of the disease, as genders show significant differences in susceptibility to severe disease and general mortality $(53,61)$. Even health authorities offering data on the age structure of the cases and deaths do not separate the age groups in the same manner (see, e.g., Tables 3-6). Important databases give only the crude case and death numbers, without further disaggregation, which might lead to misinterpretation of the true mortality differences among the countries. Moreover, this data should be merged with comorbidity-specific information to take this into account simultaneously in a sophisticated statistical analysis.

\section{Conclusions}

In conclusion, the age structure of cases, population differences regarding underlying comorbidities and risk factors, appropriate consideration of the time lag between reported infections and death, the capacity of the health system and potential underassessment of cases or deaths due to limited surveillance capacities are important to understand the differences between reported country-specific CFR estimates. Our study has also shown that further improvement towards a better coordinated and unified public health data reporting system in Europe and worldwide is highly warranted to fight this and any other pandemic that may emerge in the future.

\section{Abbreviations}

CFR: Case fatality risk

$$
\widetilde{C F R}_{j k}: \text { : Age-standardized country j on day } \mathrm{k}
$$

$d_{i j k}$ : Cumulative number of deaths for age group i, country $\mathrm{j}$, and up to day $\mathrm{k}$

$\delta_{i j k}$ : number of deaths for age group i, country j, and on day $\mathrm{k}$

$\Delta: \quad$ Lag length

ECDC: European Centre for Disease Prevention and Control

e.g.: $\quad$ exempli gratia

EU: $\quad$ European Union

hjk: $\quad$ number of hospitalizations in country j and on day $\mathrm{k}$

i.e.: $\quad$ id est

IHME: Institute for Health Metrics and Evaluation

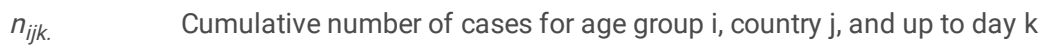

OECD: Organisation for Economic Co-operation and Development

weight of age group $i$

\section{Declarations}




\section{Ethics approval and consent to participate}

Not applicable.

\section{Consent for publication}

Not applicable.

\section{Availability of data and materials}

The datasets generated and analyzed during the current study are available from the cited sources or the corresponding author on reasonable request.

\section{Competing interests}

The authors declare that they have no competing interests.

\section{Funding}

PV, BL, and GK received funding from the European Union's Horizon 2020 research and innovation program under grant agreement No 101003480 and from the Initiative and Networking Fund of the Helmholtz Association.

\section{Authors' contributions}

$\mathrm{HB}, \mathrm{BL}$, and CW proposed the methods used in this study. PV, HB, and CW researched, organized, and structured the data and conducted the computations. BL, $\mathrm{AM}, \mathrm{AH}, \mathrm{SW}$, and $\mathrm{HB}$ did the literature research. PV and CW built the illustrations of the manuscript. All authors wrote and revised the text.

\section{Acknowledgments}

Not applicable.

\section{References}

1. World Health Organization 15 [cited 2020 Jun 12] World Health Organization. Coronavirus disease 2019 (COVID-19) Situation Report - 116. 2020 May 15 [cited 2020 Jun 12 ]. https://www.who.int/emergencies/diseases/novel-coronavirus-2019/situation-reports.

2. Lipsitch M, Donnelly CA, Fraser C, Blake IM, Cori A, Dorigatti I, et al. Potential Biases in Estimating Absolute and Relative Case-Fatality Risks during Outbreaks. PLoS Negl Trop Dis. 2015;9(7):e0003846.

3. Cai H. Sex difference and smoking predisposition in patients with COVID-19. The Lancet Respiratory Medicine. 2020;8(4):E20.

4. European Centre for Disease Prevention and Control 2020 Mar 12 [cited 2020 Apr 18]

European Centre for Disease Prevention and Control. Novel coronavirus disease 2019 (COVID-19) pandemic: increased transmission in the EU / EEA and the UK - sixth update. 2020 Mar 12 [cited 2020 Apr 18]. https://www.ecdc.europa.eu/sites/default/files/documents/RRA-sixth-update-Outbreak-of-novelcoronavirus-disease-2019-COVID-19.pdf.

5. Shim E, Tariq A, Choi W, Lee Y, Chowell G. Transmission potential and severity of COVID-19 in South Korea. Int J Infect Dis. 2020;93:339-44.

6. Wu JT, Leung K, Bushman M, Kishore N, Niehus R, de Salazar PM, et al. Estimating clinical severity of COVID-19 from the transmission dynamics in Wuhan, China. Nat Med. 2020;26:506-10.

7. Wu Z, McGoogan JM. Characteristics of and Important Lessons From the Coronavirus Disease 2019 (COVID-19). Summary of a Report of 72314 Cases From the Chinese Center for Disease Control and Prevention. JAMA. 2020;323(13):1239-42.

8. Xie J, Tong Z, Guan X, Du B, Qiu H, Slutsky AS. Critical care crisis and some recommendations during the COVID-19 epidemic in China. Intensive Care Med. 2020;46(5):837-40.

9. Yang J, Zheng Y, Gou X, Pu K, Chen Z, Guo Q, et al. Prevalence of comorbidities in the novel Wuhan coronavirus (COVID-19) infection: a systematic review and meta-analysis. Int J Infect Dis. 2020;94:91-5.

10. $10.1111 /$ all.14238 Zhang J-J, Dong X, Cao Y-Y, Yuan Y-D, Yang Y-B, Yan Y-Q, et al. Clinical characteristics of 140 patients infected with SARS-CoV-2 in Wuhan, China. Allergy. 2020 Feb 19;doi: 10.1111/all.14238.

11. Gianicolo E, Riccetti N, Blettner M, Karch A. Epidemiological Measures in the Context of the COVID-19 Pandemic. Deutsches Ärzteblatt International. 2020;117:336-42.

12. European Centre for Disease Prevention and Control. Sentinel surveillance [updated 2020 Mar 24. Available from: https://www.ecdc.europa.eu/en/seasonal-influenza/surveillance-and-disease-data/facts-sentinel-surveillance.

Page 20/26 
13. Fang Y, Zhang H, Xie J, Lin M, Ying L, Pang P, et al. Sensitivity of Chest CT for COVID-19: Comparison to RT-PCR. Radiology. $2020: 200432$.

14. Lau H, Khosrawipour V, Kocbach P, Mikolajczyk A, Ichii H, Schubert J, et al. Internationally lost COVID-19 cases. J Microbiol Immunol Infect. 2020;S16841182(20)30073-6.

15. Li R, Pei S, Chen B, Song Y, Zhang T, Yang W, et al. Substantial undocumented infection facilitates the rapid dissemination of novel coronavirus (COVID19). Science. 2020;368(6490):489-93.

16. Pan Y, Zhang D, Yang P, Poon LLM, Wang Q. Viral load of SARS-CoV-2 in clinical samples. The Lancet Infect Dis. 2020;20(4):411-2.

17. Rajgor DD, Lee MH, Archuleta S, Bagdasarian N, Quek SC. The many estimates of the COVID-19 case fatality rate. The Lancet Infect Dis. 2020. doi:10.1016/S1473-3099(20)30244-9.

18. World Health Organization. Laboratory testing for 2019 novel coronavirus (2019-nCoV) in suspected human cases. Interim guidance [updated 2020 Mar 19. Available from: https://www.who.int/publications-detail/laboratory-testing-for-2019-novel-coronavirus-in-suspected-human-cases-20200117.

19. Reich NG, Lessler J, Cummings DAT, Brookmeyer R. Estimating Absolute and Relative Case Fatality Ratios from Infectious Disease Surveillance Data. Biometrics. 2012;68(2):598-606.

20. Gordis L. Epidemiology. 5th ed. Philadelphia: Elsevier Saunders; 2014.

21. Wilson N, Kvalsvig A, Barnard LT, Baker MG. Case-Fatality Risk Estimates for COVID-19 Calculated by Using a Lag Time for Fatality. Emerg Infect Dis. 2020;26(6).

22. Rhodes A, Ferdinande P, Flaatten H, Guidet B, Metnitz PG, Moreno RP. The variability of critical care bed numbers in Europe. Intensive Care Med. 2012;38(10):1647-53.

23. Eriksson CO, Stoner RC, Eden KB, Newgard CD, Guise J-M. The Association Between Hospital Capacity Strain and Inpatient Outcomes in Highly Developed Countries: A Systematic Review. J Gen Intern Med. 2017;32(6):686-96.

24. Eurostat. Eurostat Statistics Explained: Healthcare resources statistics - beds [updated 2019 Nov 29. Available from: https://ec.europa.eu/eurostat/statistics-explained/index.php/Healthcare_resource_statistics_-_beds.

25. Ji Y, Ma Z, Peppelenbosch MP, Pan Q. Potential association between COVID-19 mortality and health-care resource availability. The Lancet Glob Health. 2020;8(4):e480.

26. Legido-Quigley H, Asgari N, Teo YY, Leung GM, Oshitani H, Fukuda K, et al. Are high-performing health systems resilient against the COVID-19 epidemic? The Lancet. 2020;395(10227):848-50.

27. Zhou F, Yu T, Du R, Fan G, Liu Y, Liu Z, et al. Clinical course and risk factors for mortality of adult inpatients with COVID-19 in Wuhan, China: a retrospective cohort study. Lancet. 2020;395(10229):1054-62.

28. Jordan RE, Adab P, Cheng KK. Covid-19: risk factors for severe disease and death - A long list is emerging from largely unadjusted analyses, with age near the top. BMJ. 2020;368:m1198.

29. World Health Organization. Assessing National Capacity for the Prevention and Control and Noncommunicable Diseases: Report of the 2019 Global Survey. Geneva: World Health Organization; 2020.

30. Onder G, Rezza G, Brusaferro S. Case-Fatality Rate and Characteristics of Patients Dying in Relation to COVID-19 in Italy. JAMA. 2020. doi:10.1001/jama.2020.4683.

31. Lipsitch M. Correspondence to: Estimating case fatality rates of COVID-19. Lancet Infect Dis. 2020. doi:10.1016/S1473-3099(20)30245-0.

32. Spychalski P, Blazynska-Spychalska A, Kobiela J. Correspondence to: Estimating case fatality rates of COVID-19. Lancet Infect Dis. 2020. doi:10.1016/S1473-3099(20)30246-2.

33. Eurostat. Population on 1 January by age and sex [updated 2020 Apr 18. Available from: https://ec.europa.eu/eurostat/data/database.

34. Robert Koch Institut. Täglicher Lagebericht des RKI zur Coronavirus-Krankheit 2019 (COVID-19). 04.03.-13.05.2020 - Aktualisierter Stand für Deutschland [updated 2020 May 13. Available from: https://www.rki.de/DE/Content/InfAZ/N/Neuartiges_Coronavirus/Situationsberichte/Archiv.html.

35. Istituto Superiore di Sanità. Studio ISS Su 105 deceduti con Covid-2019, età media 81 anni e patologie preesistenti in due terzi dei casi. Comunicati stampa 15/2020 [updated 2020 Mar 05. Available from: https://www.iss.it/en/comunicati-

stampa/-/asset_publisher/fjTKmjJgSgdK/content/id/5286166?

_com_liferay_asset_publisher_web_portlet_AssetPublisherPortlet_INSTANCE_fjTKmjJgSgdK_redirect=https\%3A\%2F\%2Fwww.iss.it\%2Fen\%2Fcomunicatistampa\%3Fp_p_id\%3Dcom_liferay_asset_publisher_web_portlet_AssetPublisherPortlet_INSTANCE_fjTKmjJgSgdK\%26p_p_lifecycle\%3D0\%26p_p_state\%3[

36. Istituto Superiore di Sanità. Letalità in Italia minore di quella della Cina per tutte le fasce di età. Comunicati stampa 16/2020 [updated 2020 Mar 06. Available from: https://www.iss.it/en/comunicati-stampa/-/asset_publisher/fjTKmjJgSgdK/content/id/5288119? _com_liferay_asset_publisher_web_portlet_AssetPublisherPortlet_INSTANCE_fjTKmjJgSgdK_redirect=https\%3A\%2F\%2Fwww.iss.it\%2Fen\%2Fcomunicatistampa\%3Fp_p_id\%3Dcom_liferay_asset_publisher_web_portlet_AssetPublisherPortlet_INSTANCE_fjTKmjJgSgdK\%26p_p_lifecycle\%3D0\%26p_p_state\%3[

37. Istituto Superiore di Sanità. Sorveglianza Integrata COVID-19 in Italia. AGGIORNAMENTO 11 marzo 2020 [updated 2020 Mar 11. Available from: https://www.epicentro.iss.it/coronavirus/sars-cov-2-sorveglianza-dati.

38. Istituto Superiore di Sanità. Epidemia COVID-19. Aggiornamento nazionale. 19 marzo - 14 maggio 2020 - ore 16:00 [updated 2020 May 15. Available from: https://www.epicentro.iss.it/coronavirus/sars-cov-2-sorveglianza-dati.

39. Santé publique France. COVID-19: Point épidémiologique - Situation au 4-15 mars 2020 à minuit [updated 2020 Mar 15. Available from: https://www.santepubliquefrance.fr/recherche/\#search=COVID-19\%20:\%20point\%20\%C3\%A9pid\%C3\%A9miologique.

40. Santé publique France. COVID-19: Point épidémiologique hebdomaire du 24 mars - 14 mai 2020 [updated 2020 May 14. Available from: https://www.santepubliquefrance.fr/recherche/\#search=COVID-19\%20:\%20point\%20\%C3\%A9pid\%C3\%A9miologique.

Page $21 / 26$ 
41. Instituto de Salud Carlos III. Informe sobre la situación de COVID-19 en España. Informe COVID-19 no 6-32. 05 de marzo - 21 de mayo de 2020 [updated 2020 May 21. Available from:

https://www.isciii.es/QueHacemos/Servicios/VigilanciaSaludPublicaRENAVE/EnfermedadesTransmisibles/Paginas/InformesCOVID-19.aspx.

42. Gesundheitsberichtserstattung des Bundes. Im Informationssystem der GBE zur Altersstandardisierung benutzte Standardbevölkerungen. Gliederungsmerkmale: Alter, Geschlecht, Standardbevölkerung [updated 2020 Apr 17. Available from: http://www.gbe-bund.de/.

43. European Centre for Disease Prevention and Control. Download today's data on the geographic distribution of COVID-19 cases worldwide [updated 2020 Jun 15. Available from: https://www.ecdc.europa.eu/en/publications-data/download-todays-data-geographic-distribution-covid-19-cases-worldwide.

44. OECD

OECD. Beyond Containment. Health systems responses to COVID-19 in the OECD [updated 2020 Apr 16. Available from: https://read.oecdilibrary.org/view/?ref=119_119689-ud5comtf84\&Title=Beyond\%20Containment:Health\%20systems\%20responses\%20to\%20COVID19\%20in\%20the\%200ECD.

45. Institute for Health Metrics and Evaluation. COVID-19 Projections: Germany Seattle: Institute for Health Metrics and Evaluation; [updated 2020 May 292020 Apr 29]. Available from: https://covid19.healthdata.org/germany.

46. Institute for Health Metrics and Evaluation. COVID-19 Projections: Italy Seattle: Institute for Health Metrics and Evaluation; [updated 2020 May 292020 Apr 29]. Available from: https://covid19.healthdata.org/italy.

47. Institute for Health Metrics and Evaluation. COVID-19 Projections: France Seattle: Institute for Health Metrics and Evaluation; [updated 2020 May 292020 Apr 29]. Available from: https://covid19.healthdata.org/france.

48. Institute for Health Metrics and Evaluation. COVID-19 Projections: Spain Seattle: Institute for Health Metrics and Evaluation; [updated 2020 May 292020 Apr 29]. Available from: https://covid19.healthdata.org/spain.

49. Verity R, Okell LC, Dorigatti I, Winskill P, Whittaker C, Imai N, et al. Estimates of the severity of coronavirus disease 2019: a model-based analysis. Lancet Infect Dis. 2020. doi:10.1016/S1473-3099(20)30243-7.

50. IHME COVID-19 health service utilization forecasting team. Forecasting the impact of the first wave of the COVID-19 pandemic on hospital demand and deaths for the USA and European Economic Area countries. medRxiv. 2020; doi: 10.1101/2020.04.21.20074732.

51. 51.

52. Contini D, Costabile F. Does Air Pollution Influence COVID-19 Outbreaks? Atmosphere. 2020;11(4):377.

53. Vanella P. A principal component model for forecasting age- and sex-specific survival probabilities in Western Europe. Zeitschrift für die gesamte Versicherungswissenschaft. (German Journal of Risk Insurance). 2017;106(5):539-54.

54. Zhao S, Musa SS, Lin Q, Ran J, Yang G, Wang W, et al. Estimating the Unreported Number of Novel Coronavirus (2019-nCOV) Cases in China in the First Half of January 2020: A Data-Driven Modelling Analysis of the Early Outbreak. Journal of Clinical Medicine. 2020;9(2):388.

55. Stringhini S, Wisniak A, Piumatti G, Azman AS, Lauer SA, Baysson H, et al. Seroprevalence of anti-SARS-CoV-2 IgG antibodies in Geneva, Switzerland (SEROCoV-POP): a population-based study. The Lancet. 2020. doi:10.1016/S0140-6736(20)31304-0.

56. Istituto Superiore di Sanità. Sorveglianza Integrata COVID-19 in Italia. AGGIORNAMENTO 08 aprile 2020 [updated 2020 Apr 08. Available from: https://www.epicentro.iss.it/coronavirus/sars-cov-2-sorveglianza-dati.

57. Tuite AR, Ng V, Rees E, Fisman D. Estimation of COVID-19 outbreak size in Italy. Lancet Infect Dis. 2020;20(5):537.

58. Mizumoto K, Kagaya K, Zarebski A, Chowell G. Estimating the asymptomatic proportion of coronavirus disease 2019 (COVID-19) cases on board the Diamond Princess cruise ship, Yokohama, Japan, 2020. Eurosurveillance. 2020;25(10):2000180.

59. Johns Hopkins University. COVID-19 Dashboard by the Center for Systems Science and Engineering (CSSE) at Johns Hopkins University (JHU) [updated 2020 Jun 172020 Jun 17]. Available from:

https://gisanddata.maps.arcgis.com/apps/opsdashboard/index.html\#/bda7594740fd40299423467b48e9ecf6.

60. World Health Organization. Coronavirus disease 2019 (COVID-19) Situation Report - 46 [updated 2020 March 06. Available from: https://www.who.int/emergencies/diseases/novel-coronavirus-2019/situation-reports.

61. Spagnolo PA, Manson JE, Joffe H. Sex and Gender Differences in Health: What the COVID-19 Pandemic Can Teach Us. Ann Intern Med. 2020. doi:10.7326/M20-1941.

\section{Figures}




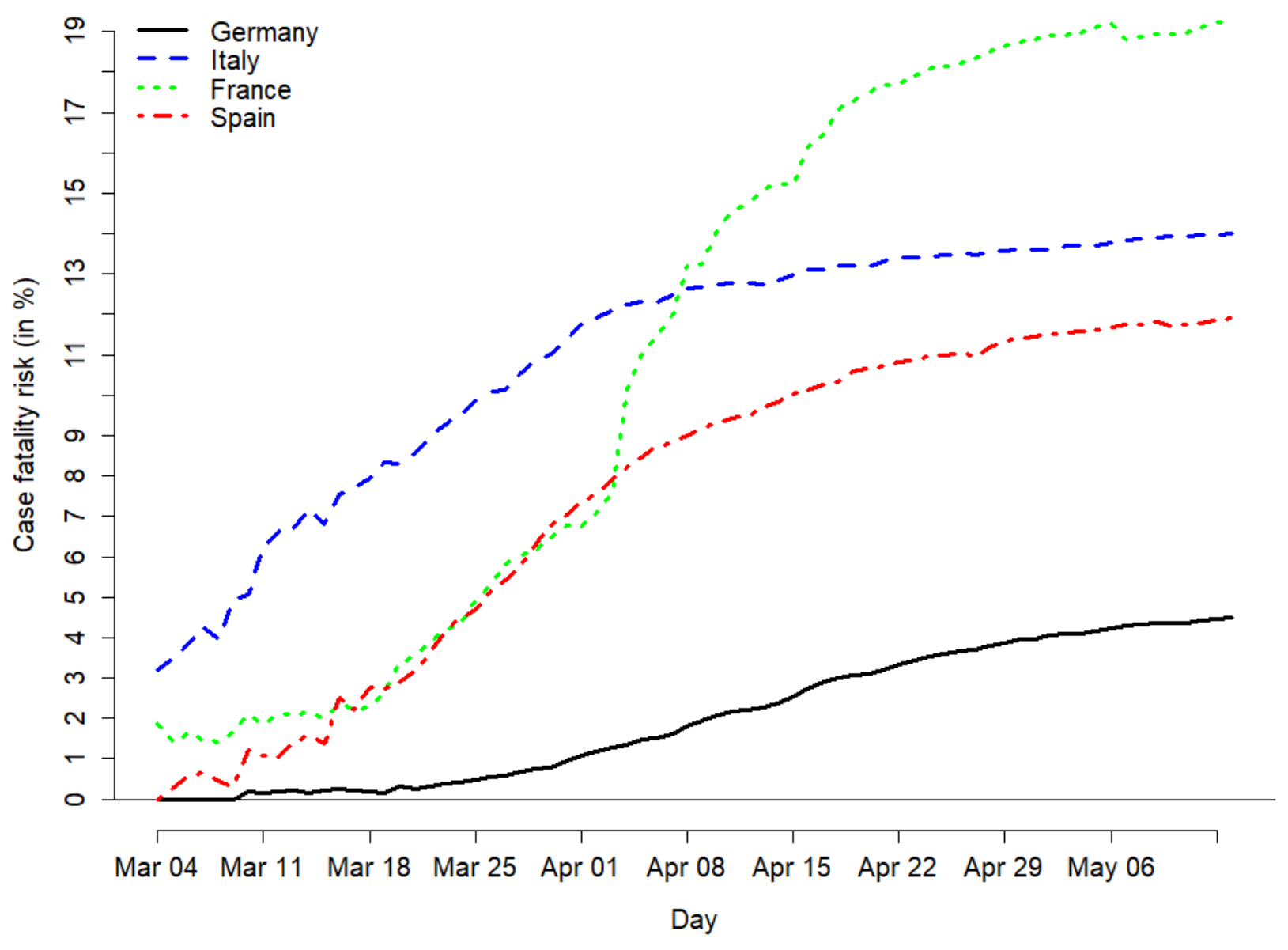

Figure 1

Crude Case Fatality Risk Estimates due to COVID-19 between March 4th and May 14th, 2020 Germany Italy France Spain Sources: (43); Own computation and design 


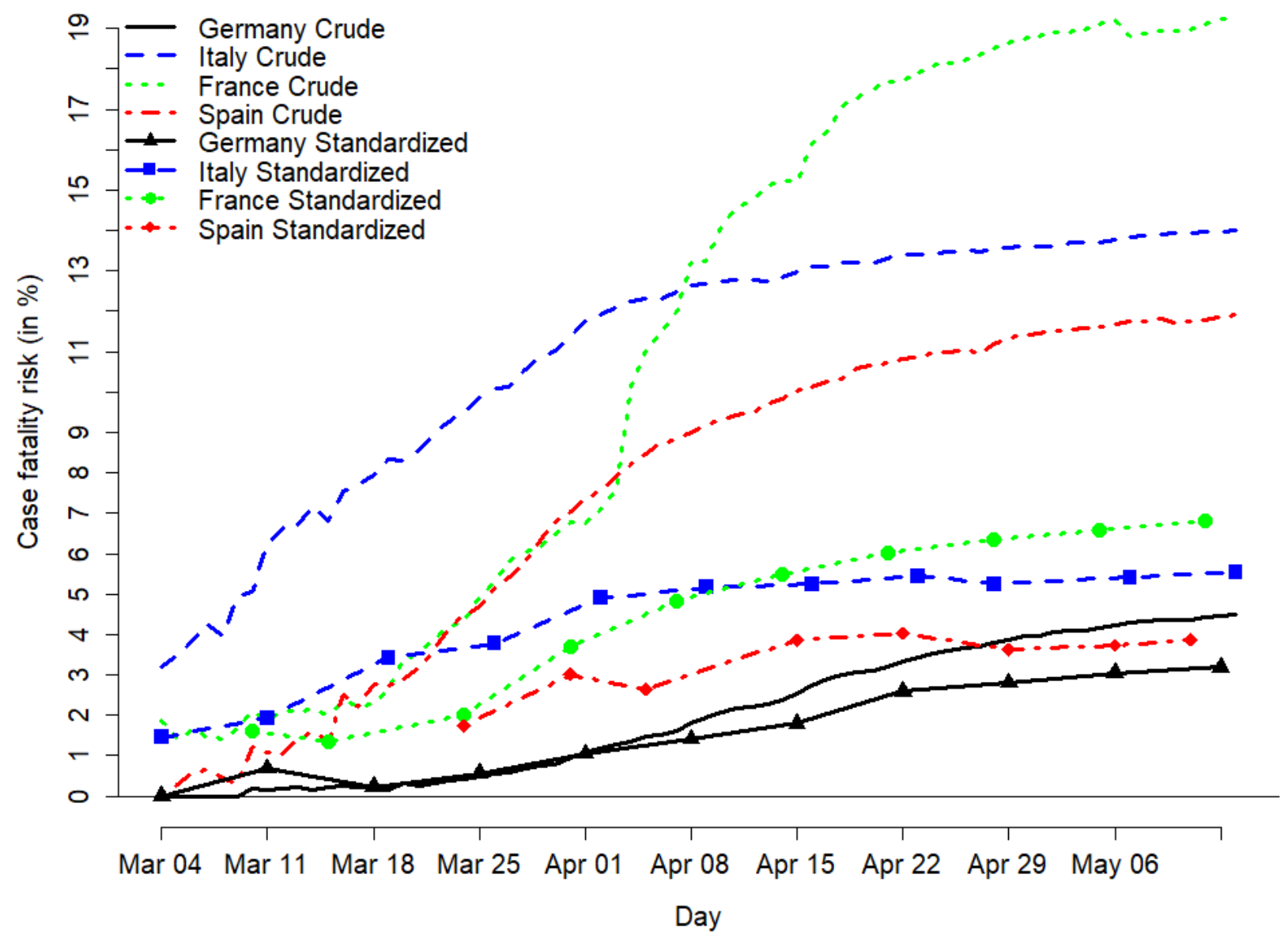

Figure 2

Crude and age-standardized CFR estimates Germany Crude Italy Crude France Crude Spain Crude Germany Standardized Italy Standardized France Standardized Spain Standardized Sources: (34-42); own computation and design 
Germany

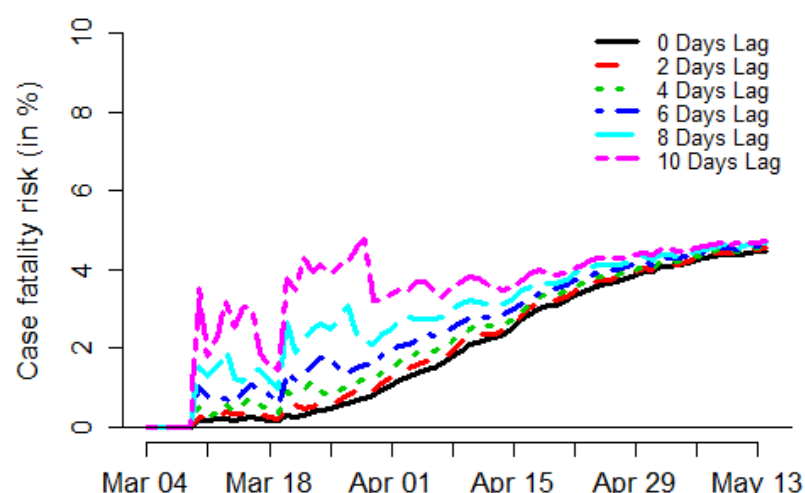

Day

France

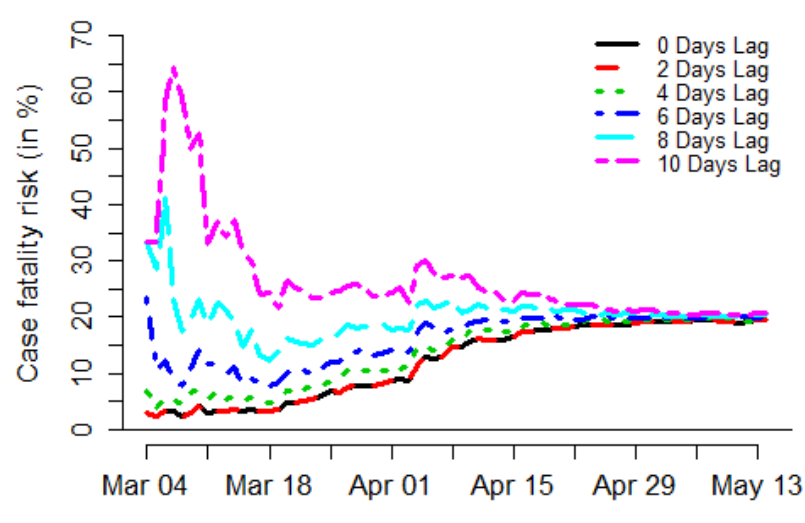

Day
Italy

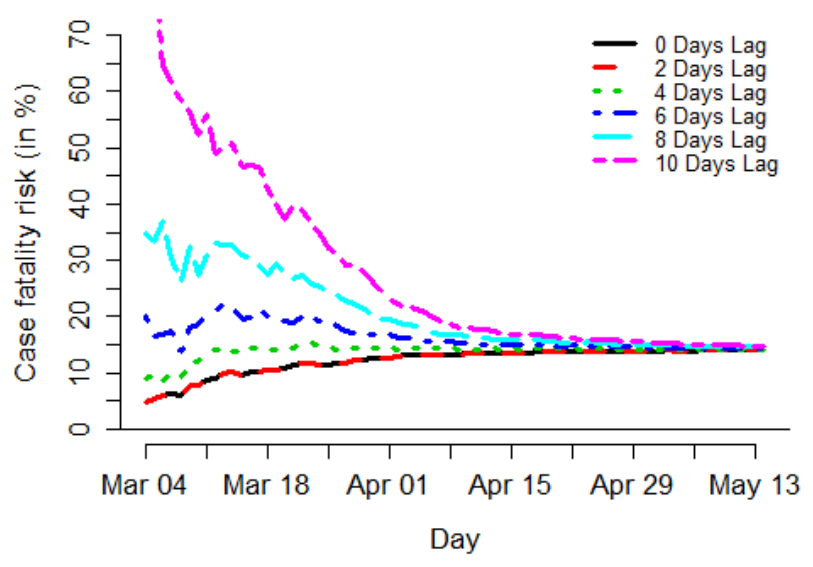

Spain

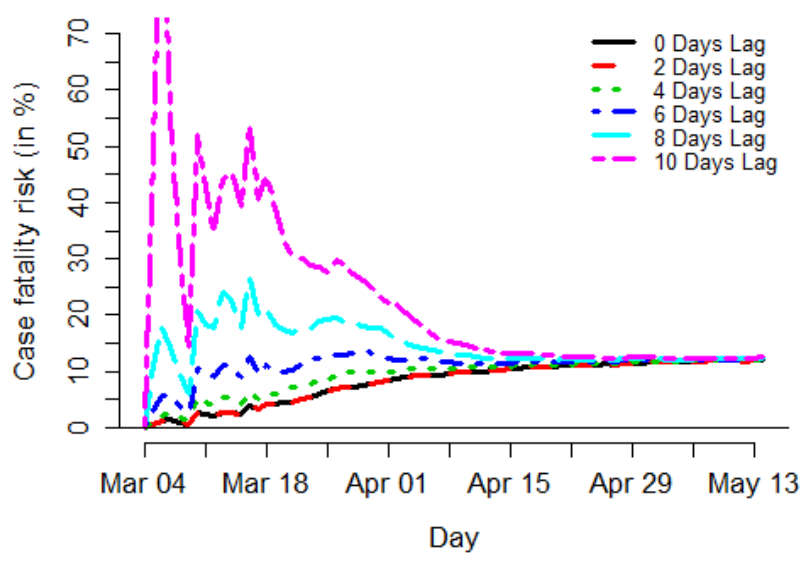

Figure 3

Crude CFR estimates for Germany, Italy, France and Spain from March 4th, 2020 until May 14th, 2020, with different time lags 0 Days Lag 2 Days Lag 4 Days Lag 6 Days Lag 8 Days Lag 10 Days Lag Sources: (43); Own computation and design 


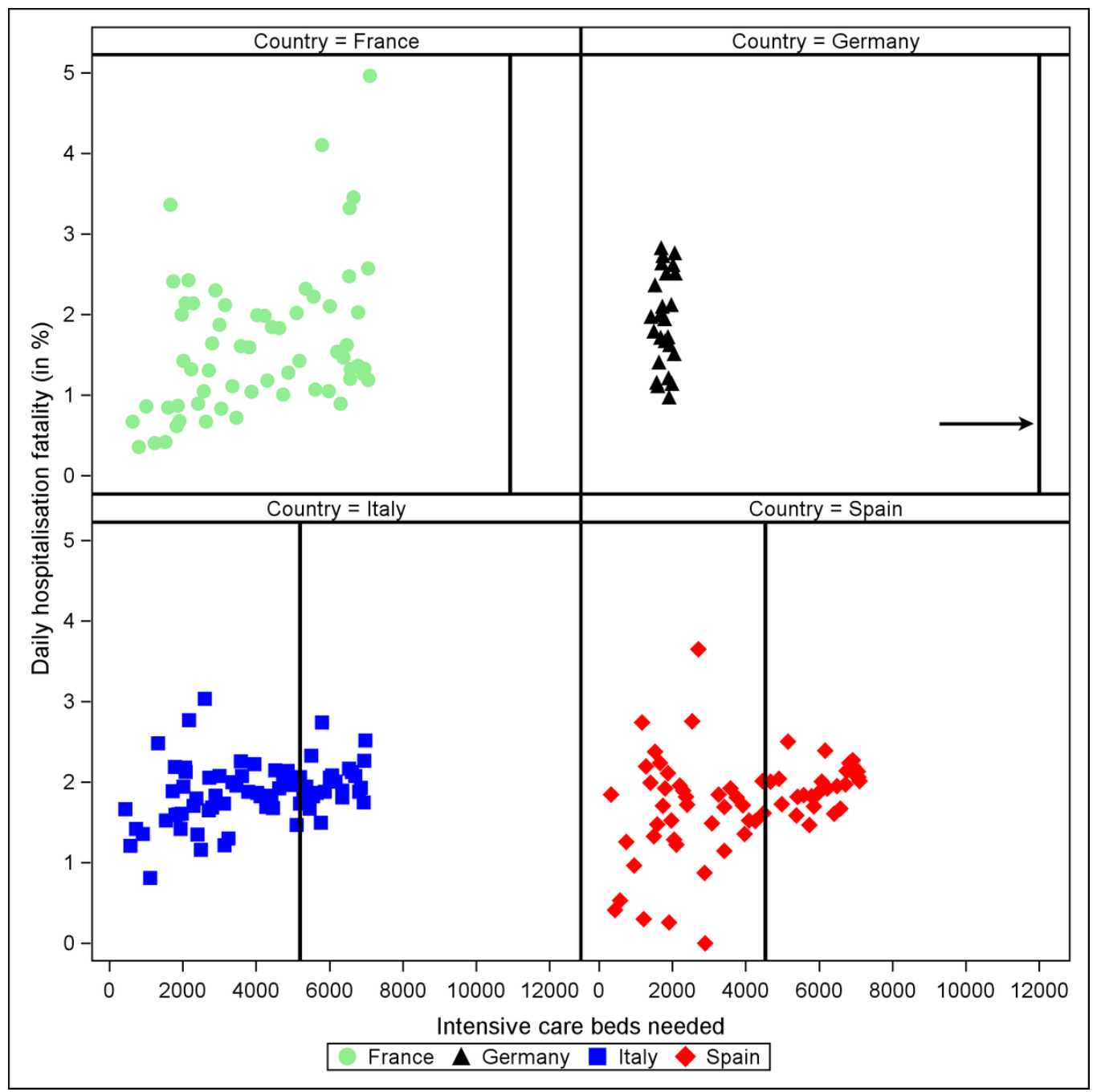

Figure 4

Daily hospitalization fatality relative to intensive care beds needed for COVID-19 patients France Germany Italy Spain Sources: (43-48); Own computation and design 